PALEO

Revue d'archéologie préhistorique

19 | 2007

Spécial table ronde (1 ère partie) : Le Gravettien : entités régionales d'une paléoculture européenne, Les Eyzies, juillet 2004

\title{
Le Gravettien du nord des Carpathes et des Sudètes
}

Gravettian North of the Carpathians and of the Sudetes

Janusz K. Kozlowski

\section{OpenEdition}

Journals

Édition électronique

URL : https://journals.openedition.org/paleo/620

DOI : $10.4000 /$ paleo.620

ISSN : 2101-0420

Éditeur

SAMRA

Édition imprimée

Date de publication : 30 décembre 2007

Pagination : 221-241

ISSN : $1145-3370$

Référence électronique

Janusz K. Kozlowski, "Le Gravettien du nord des Carpathes et des Sudètes », PALEO [En ligne], 19| 2007, mis en ligne le 23 avril 2009, consulté le 27 juin 2021. URL : http://journals.openedition.org/ paleo/620 ; DOI : https://doi.org/10.4000/paleo.620

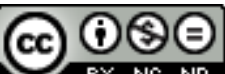

PALEO est mis à disposition selon les termes de la licence Creative Commons Attribution - Pas d'Utilisation Commerciale - Pas de Modification 4.0 International. 


\title{
LE GRAVETTIEN \\ DU NORD DES CARPATHES ET DES SUDETES
}

\author{
Janusz K.KOZLOWSKI ${ }^{(1)}$
}

\begin{abstract}
Résumé : La zone nord des Carpates et des Sudètes a été sporadiquement pénétrée, entre 26 et 24 Kyr BP, avant le maximum du Pléniglaciaire (LGM), par des groupes de chasseurs pavloviens du bassin moyen du Danube. Les preuves des migrations saisonnières systématiques entre les deux versants des Carpates n'apparaissent que dans l'horizon récent du Gravettien à pointes à cran. Ces groupes ont émigré vers l'Est au maximum du Pléniglaciaire, contribuant à l'origine du Kostenkien. La zone occidentale, à l'ouest de I'Oder, n'était que très rarement pénétrée par les groupes gravettiens provenant plutôt de l'Ouest. Les chasseurs gravettiens du bassin supérieur du Dnester et de la Volhynie restent en relation avec le Molodovien du bassin moyen du Dniester.
\end{abstract}

Mots-clés : Gravettien, Pavlovien, Molodovien, horizon à pointes à cran, Maximum du Pléniglaciaire (LGM), chasseurs de mammouths.

Abstract: Gravettian North of the Carpathians and of the Sudetes. The zone North of the Carpathians and the Sudetes was only sporadically penetrated by Gravettian hunters of the middle Danube Pavlovian between 26 and 24 Kyr B.P. Evidence of systematic seasonal migrations between southern and northem slopes of the Carpathians appears only between 24 and 22 Kyr B.P., in the shouldered point horizon, before the LGM, when Late Gravettian groups emigrated from Central to Eastern Europe. This migrations contributed to the formation of the Kostenki-Avdeevo Culture. The zone west of the Oder was only sporadically penetrated, mostly by Gravettian groups from Western Europe. The teritory of the Uppper Dnester Basin and Volhynia belongs to the area of the Molodovian from middle Dnester basin.

Key words: Gravettian, Pavlovian, Molodovian, shouldered point horizon, LGM, Mammoth hunters.

\section{INTRODUCTION}

Le but de cet article est de présenter l'évolution du Gravettien sur les plateaux loessiques de l'Europe moyenne, dans les bassins supérieurs du Dniester, de la Vistule, de l'Oder et de l'Elbe. Ces terrains sont subdivisés entre l'Ukraine occidentale, la Pologne méridionale et l'est de l'Allemagne. Trois zones éco-géographiques ont été distinguées sur ce territoire : la zone orientale qui comprend le bassin supérieur du Dniester et la Volhynie, la zone centrale des bassins supérieurs de la Vistule et de l'Oder et la zone occidentale des bassins de l'Elbe et de Saale (fig.1). Nous allons présenter successivement les changements de milieu naturel dans la période située entre 30 et $15 \mathrm{Kyr} \mathrm{BP}$, l'évolution de l'habitat, des stratégies de subsistance et de la culture matérielle du Gravettien dans ces trois zones.

\section{Zone orientale}

Bases chronostratigraphiques et changements de I'environnement (tabl. 1)

La meilleure séquence stratigraphique dans le bassin de Dniester a été mise au jour dans le site de Molodova V. Les résultats des premières observations (Chernysh 1959, 1987 ; Ivanova et Tseitlin 1987) ont été ré-évaluées par P. Haesaerts (et al. 2004).

Dans la période située entre 33 et $27 \mathrm{Kyr}$, un complexe pédologique s'est formé ; il se compose de deux sols bruns du type para-rendzina, notamment 10/1 (32,6 - 30,4 Kyr BP) et 10/2 (28,7-27,7 Kyr BP). Les premiers horizons gravettiens (10 et 9) correspondent a l'épisode froid entre les deux oscillations tempérées. 


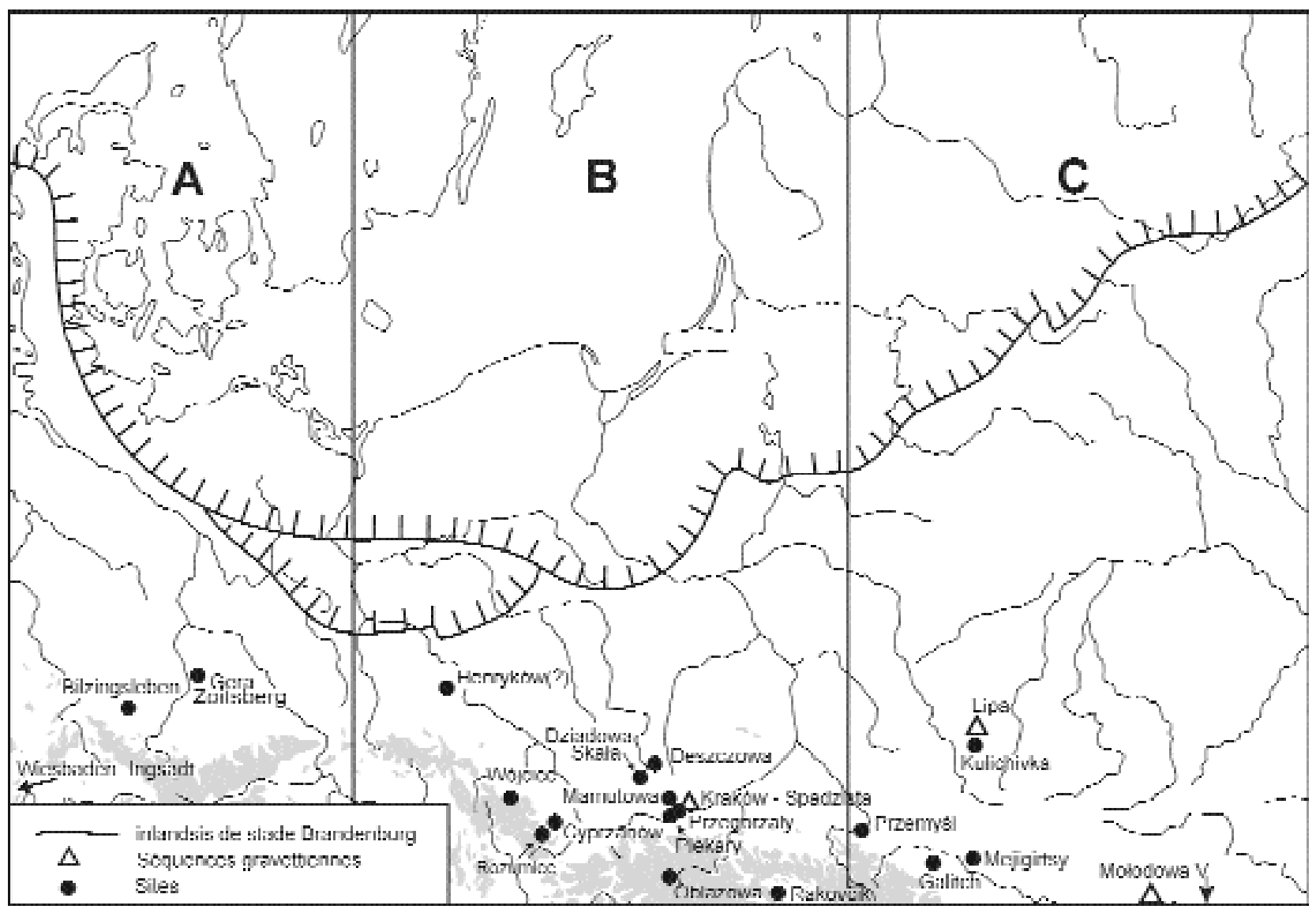

Figure 1 - Carte de trois zones éco-géographiques discutées dans cet article.

Figure 1 - Map of the three eco-geographical zones discussed in this paper.

Les paléosols du complexe 10 sont superposés par un horizon humifère (11/2) qui a fourni les témoignages du Gravettien moyen (niveau d'habitat 8). Ce niveau archéologique n'a pas fourni de

Par contre, le sommet loessique de la couche $11(11 / 3)$ a donné des datations absolues entre 25,2 - 25,1 Kyr BP, I'horizon humifère sus-jacent (unité 12/1) la datation 23.6 Kyr et le gley de toundra (unité 12/2) l'âge de 23,0 Kyr BP. Tout ce complexe de couches sédimentées dans des conditions climatiques variables a fourni plusieurs horizons de traces d'habitats du Gravettien supérieur à pointes à cran, bien qu'il soit difficile d'identifier la position de l'habitat principal de la couche 7, fouillée par A.P. Tchemysh.

La couche 13 de Molodova $V$ reflète des conditions de plus en plus sèches avec apports loessiques entre 23,0 et 20,0 Kyr BP, avec un seul épisode moins froid autour de 21,5 Kyr $\mathrm{BP}$, permettant la formation d'un sol initial bioturbé. Cette période n'a pas fourni de traces d'habitat gravettien.

La période de la transgression maximale de l'inlandsis entre 20,0 et 17,0 Kyr BP fut plus humide, mais toujours avec dépôt de loess, parfois avec des épisodes de gel profond (unité stratigraphique 14, partie inférieure) ; elle se ter- mine par un sol humifère. Plus tard, entre 17,0 et $14,5 \mathrm{Kyr}$ $\mathrm{BP}$ les conditions de permafrost et une sédimentation éolienne intense réapparaissent (partie supérieure de l'unité 14). Pendant cette période, des occupations epigravettiennes (6 a 4) ont eu lieu.

L'évolution de l'environnement avait sans doute influencé la composition de la faune chassée par les groupes gravettiens dans la vallée du Dniester. Si dans le Gravettien ancien, le Cheval est plus abondant que le Renne, dans le Gravettien moyen la proportion entre les restes de ces animaux est la même, mais le Mammouth joue un rôle important comme dans le Gravettien récent. Dans l'Epigravettien le Renne devient le gibier préféré, et le Mammouth disparaît à partir de la couche 4 , remplacé par le Bison.

Les séquences stratigraphiques du bassin du Dniester supérieur sont beaucoup moins complétes. Les sites avec un enregistrement sédimentaire et archéologique continu sont rares, aussi bien pendant le Gravettien que pendant l'Épigravettien, comme les sites du Dniester moyen.

Une étude dans la région de Galitch a été publiée récemment sous la direction de T. Madeyska (2002). La séquence 


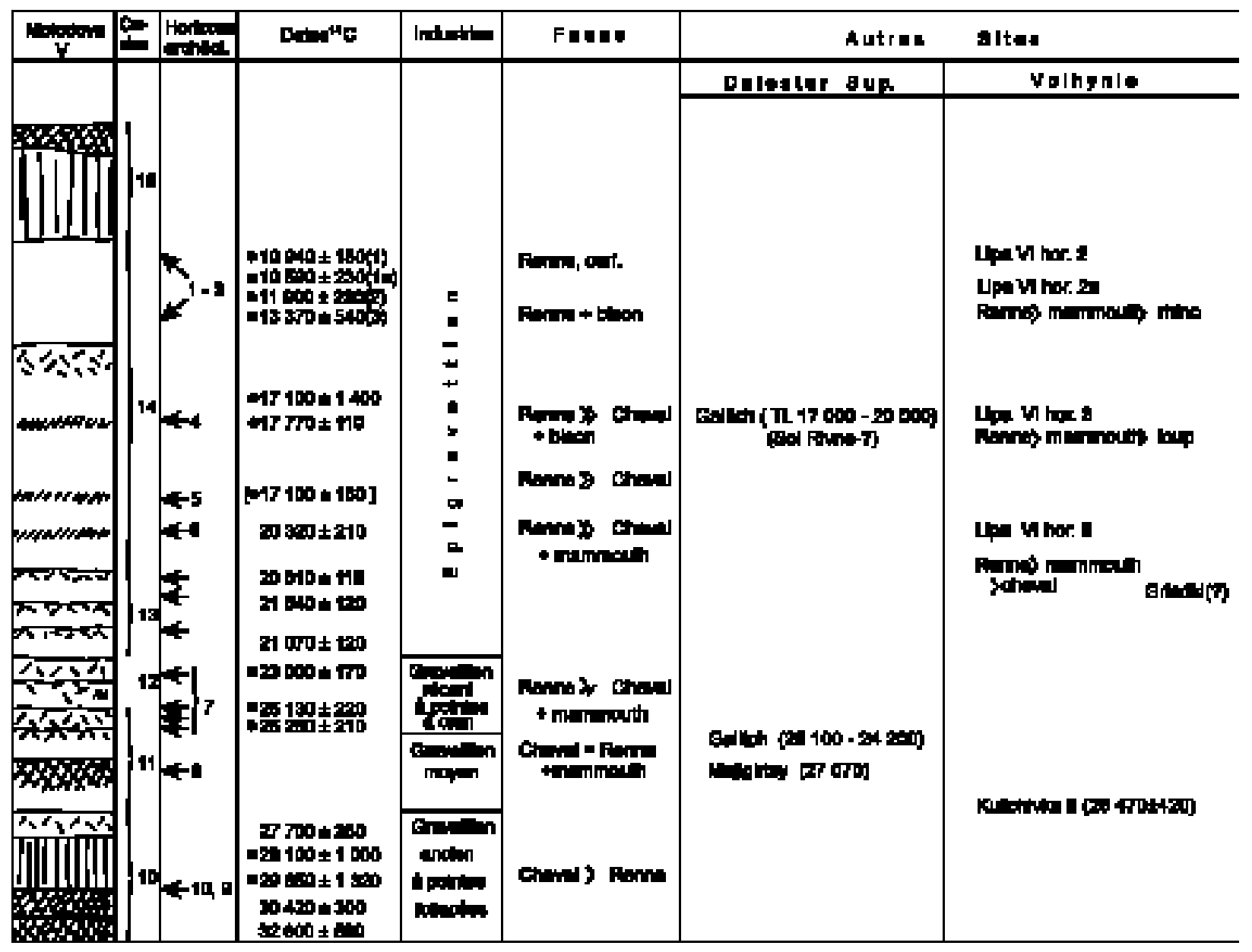

Tableau 1 - Séquence de Molodova V comparée aux sites du bassin supérieur du Dniester et de la Volhynie.

Table 1 - Molodova V sequence compared to the Gravettian sites in the upper Dniester.

enregistrée dans les coupes de Galitch I a fourni au moins trois niveaux d'habitats situés au-dessous du paléosol, appelé "sol de Rivné" (Bogutskiy 1986) que l'on peut corréler avec le "sol de Dofinovka" (Gozhik et al. 2001), correspondant probablement à l'oscillation de Lascaux. En effet, dans les séquences du Dniester supérieur, on note la présence de deux paléosols post-Pléniglaciaires (de Rivne/Dofinovka et de Krasyliv). Dans ces conditions d'enregistrement sédimentaire incomplet du cycle climatique, la datation des niveaux archéologiques est basée presque uniquement sur les méthodes radiométriques, ce qui pourrait conduire à des ambigüités. Les trois niveaux gravettiens de Galitch I sont datés par ${ }^{14} \mathrm{C}$ au Laboratoire de Kiev entre 25 $100 \pm 450$ et $23500 \pm 600$ BP mais en même temps, les datations TL pour les échantillons du loess du niveau inférieur sont 18,1 et 17,9 $\pm 2,4-2,6$ ce qui reporterait l'habitat de Galitch I à l'Epigravettien (Madeyska ed. 2002).

Un autre site gravettien important, Mejigirtsy, est situé sur la rive gauche du Dniester (Klaptchuk 1983 ; Koulakovskaya 1989). La position chrono-stratigraphique des occupations gravettiennes dans ce site a été corrélée par M. Lanczot et A. Bogutskiy (2002) avec la partie supérieure humique du paléosol subarctique bipartite brun, attribué au sol de Rivne. Les datations ${ }^{14} \mathrm{C}$ obtenues dans le Laboratoire de Kiev se situent entre $17200 \pm 250$ et 17560 \pm 270 BP. Par contre, la datation ${ }^{14} \mathrm{C}$ AMS de Oxford est plus ancienne (20 $360 \pm 200 \mathrm{BP})$ (Koulakovska et Otte 1998). Notons que P. Haesearts (et al. 2004) fait état d'une datation encore plus ancienne pour Mejigirtsy : $27070 \mathrm{BP}$. Tout cela nous conduit à la conclusion que les auteurs de l'étude des sites du Dniester supérieur, publiée en 2002 (Madeyska et al. 2002) avaient trop simplifié les séquences loessiques de cette région et, par conséquent, les seules séquences de référence sont celles que $P$. Haesearts avait publiées de la zone du Dniester moyen.

Notons que la faune des deux sites en question est différente : si à Galitch I les restes de Mammouth dominent (la presque totalité des ossements provenant de l'horizon inférieur - Wojtal et al. 2001) à Mejigirtsy, le Renne occupe la première place sur la liste de la faune, d'ailleurs plus differenciée (Cheval, Mammouth, Ours brun, Renard, Lemming). 
Nous avons encore moins de données pour corréler les séquences de la Volhynie. La coupe de Lipa VI (Savitch 1975) montre, parallèlement à l'enregistrement archéologique et sédimentaire du site de Molodova V, plusieurs phases d'occupations épigravettiennes mais sans données radiométriques.

\section{Evolution du Gravettien/Epigravettien}

L'évolution culturelle du bassin du Dniester et celle de la Volhynie sont comparables, sauf dans leur phase ancienne qui se rapproche du Gravettien du bassin carpathique. Datée entre 31 000/30 000 et 27 000/26 000 BP, elle est caractérisée par la présence des pointes foliacées (niveaux X, IX de Molodova V), avec lamelles à dos et grandes lames appointées (Chernish 1961, 1973). Cette phase est inconnue dans le bassin supérieur du Dniester, mais bien représentée en Moldavie (Borziak et al. 1981). Elle serait l'équivalent chronologique de la phase à fléchettes dans le bassin Danubien (Otte et Noiret 2004).

La phase moyenne du Gravettien - datée entre 26 et 24 Kyr BP - est connue dans le Dniester moyen par la couche VIII de Molodova V, caractérisée par les burins dièdres, lames retouchées et trés rares lamelles à dos. Les sites du bassin supérieur du Dniester, que nous pouvons éventuellement attribuer au Gravettien moyen, comme Mejigirtsy et Galitch I, montrent par contre une bonne fréquence de pièces a dos. A Mejigirtsy I, ce sont des pointes élancées à dos presque rectiligne, des lamelles à dos denticulées, des troncatures microlithiques et des ébauches de lamelles à dos (fig. 2 - Sitnik et al. 1996 ; Koulakovskaya et Otte 1998). La technologie de production laminaire dans ce site est trés proche des outillages de la partie moyenne de Molodova V. Le site de Galitch I a fourni également un bon nombre de pièces à dos, particulièrement des pièces microlithiques : lames à dos rectiligne, lamelles à dos, parfois avec amincissement basal, lamelles à deux dos et lamelles tronquées (fig.3). Le débitage laminaire est proche du Molodovien (Madeyska, ed. 2002, fig. 85-87). Les outillages en question sont donc, du point de vue stylistique, plutôt proches du Pavlovien à éléments à dos microlithiques et paragéométriques (Svoboda 2004, p. 286) que du Molodovien mais technologiquement, leur débitage laminaire ressemble plutôt au débitage laminaire molodovien.

Nous pouvons éventuellement attribuer le niveau supérieur de site Koulitchivka en Volhynie à la phase moyenne du Gravettien (Savitch 1975), surtout en raison de la datation ${ }^{14} \mathrm{C}$ de $26740 \pm 420$ BP. L'industrie lithique, avec grandes lames retouchées et appointées et de rares pièces à dos (fig. 4), ressemble plutôt à celle de la couche 8 de Molodova V. La faune est dominée par le Renne et le Mammouth, bien que le Cheval soit aussi représenté.

Le Gravettien récent, avec les pointes à cran, est bien connu par la couche 7 de Molodova V. Les examens stratigraphiques détaillées de P. Haesaerts (et al. 2004) montrent qu'il s'agit d'un complexe de plusieurs niveaux d'habitat eparpillés dans trois unités lithostratigraphiques (du sommet de 11 a 13). Paul Haesaerts suppose que l'occu- pation avec pièces à cran la plus importante se situe entre 25230 et 25130 BP et qu'elle est plus ancienne que l'occupation trouvée dans l'horizon humifère (12/2) et datée de 23650 - 23000 BP. Néanmoins, la question reste ouverte puisque pendant les fouilles de P. Haesaerts, les pièces lithiques diagnostiques n'ont pas été trouvées.

Dans le bassin du Dniester supérieur et en Volhynie, aucun site Gravettien à pointes à cran n'a été trouvé. Notons cependant qu'il existe un site-fantôme dans la vallée de San près de Przmysl (ville sur la frontière entre la Pologne et l'Ukraine) qui a été fouillé par un amateur dans les années 1930. La matériel de ce site a été égaré pendant la deuxième guerre mondiale, mais d'après les photos, il y avait une industrie en os évoquant les objets de la culture de Kostenki-Avdeevo (Gravettien oriental à pointes à cran).

Pendant la période située entre 23000 et 20000 BP, I'habitat est beaucoup moins dense dans le bassin du Dniester et en Volhynie (Kozlowski 1996 ; Djindjian 2002), probablement en raison des conditions xérothemiques de la sédimentation dans cette zone de la seconde génération du loess récent supérieur. Dans la séquence de Molodova $V$, nous pouvons éventuellement attribuer à cette période la couche archéologique 6 , datée environ de 20 Kyr BP Elle correspond à la transition entre le Gravettien et l'Epigravettien.

Le bassin du Dniester moyen et supérieur, dans la période entre 20000 et 17000 ans BP, correspondant au maximum du Pléniglaciaire supérieur (stade de BrandenbourgLeszno), n'a été que rarement occupé par les chasseurs de rennes dont les refuges se trouvaient plus au sud, en Moldavie (Otte et Noiret 2004).

Le début de l'Epigravettien en Volhynie, bien connu par la séquence de Lipa VI (Savitch 1975), se situerait autour de 17 Kyr BP. Les couches 5-2 de Lipa VI présentent deux types de débitage laminaire. Le premier, caractéristique de la couche 5 , avec une chaîne opératoire comportant des nucléus assez courts à un plan de frappe avec débitage semi-tournant presque sans préparation préliminaire (bien que des lames à crête existent Noiret 2003), mais avec des plans de frappe réavivés par des enlèvements centripètes. De cette chaîne opératoire, sont issues des lames assez courtes et peu régulières. Ce débitage diffère de celui de la séquence de Molodova V. Par contre, la couche 5 de Lipa VI a fourni des manches d'outils lithiques, en os, avec rainure latérale, typique du Molodovien (Savitch 1975, fig. 22). A partir de la couche archéologique 3, le débitage laminaire comporte des nucléus plus grands, avec des plans de débitage latéraux (Savitch 1975, fig. 25 : 4,6). Ces nucléus ont été parfois, dans leur phase finale d'exploitation, transformés en nucléus multidirectionels à éclats. Le plein débitage laminaire fournissait des lames trés régulières, parfois supérieures à $10 \mathrm{~cm}$, transformées essentiellement en burins dièdres (fig. 5). Par contre, le taux de lamelles à dos est trés bas, comme dans certaines couches de la séquence de Molodova V. 


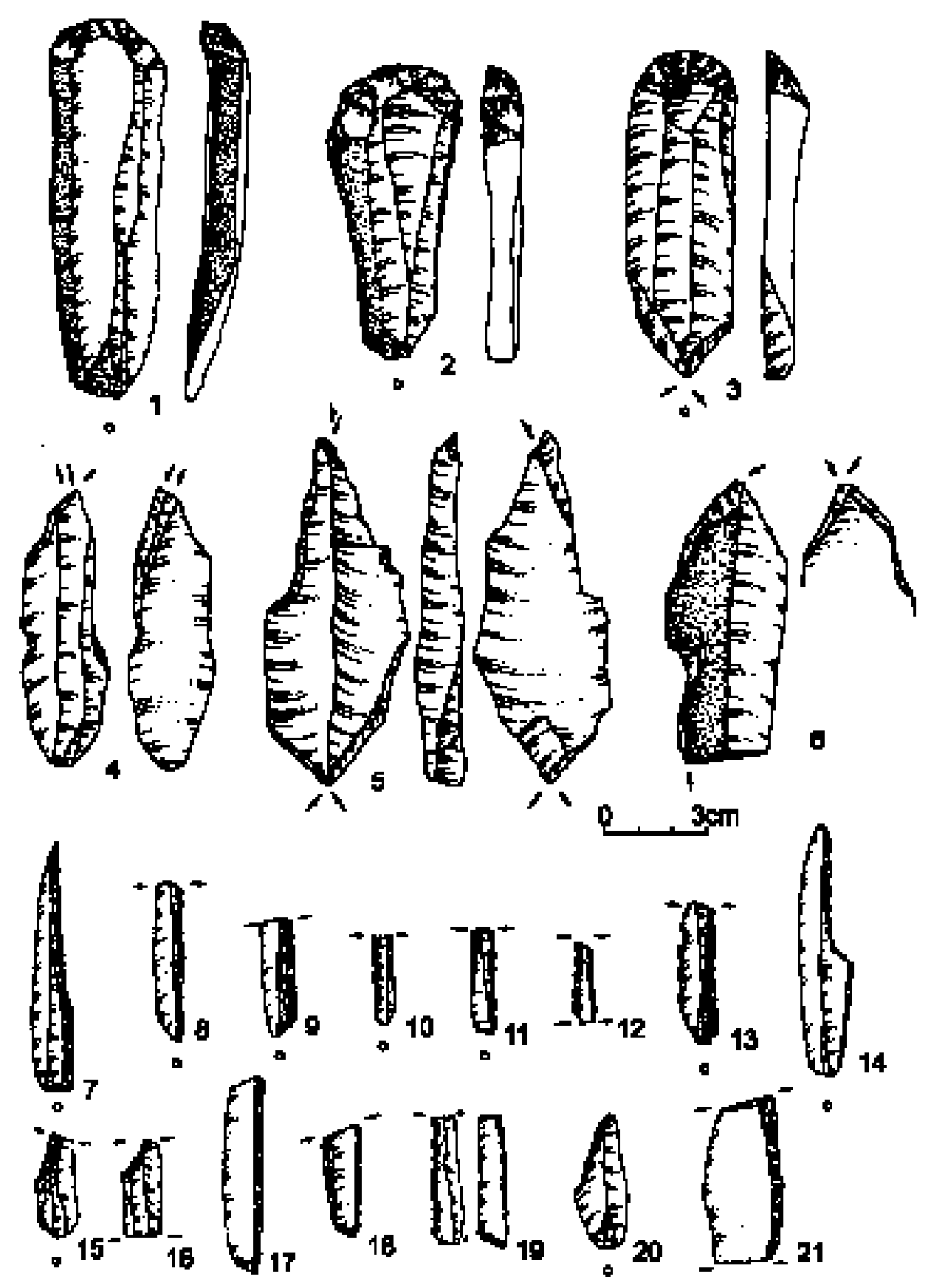

Figure 2 - Mejigirtsy (Ukraine occidentale). Lamelles à dos.

Figure 2 - Mejigirtsy (western Ukraina). Backed bladelets. 

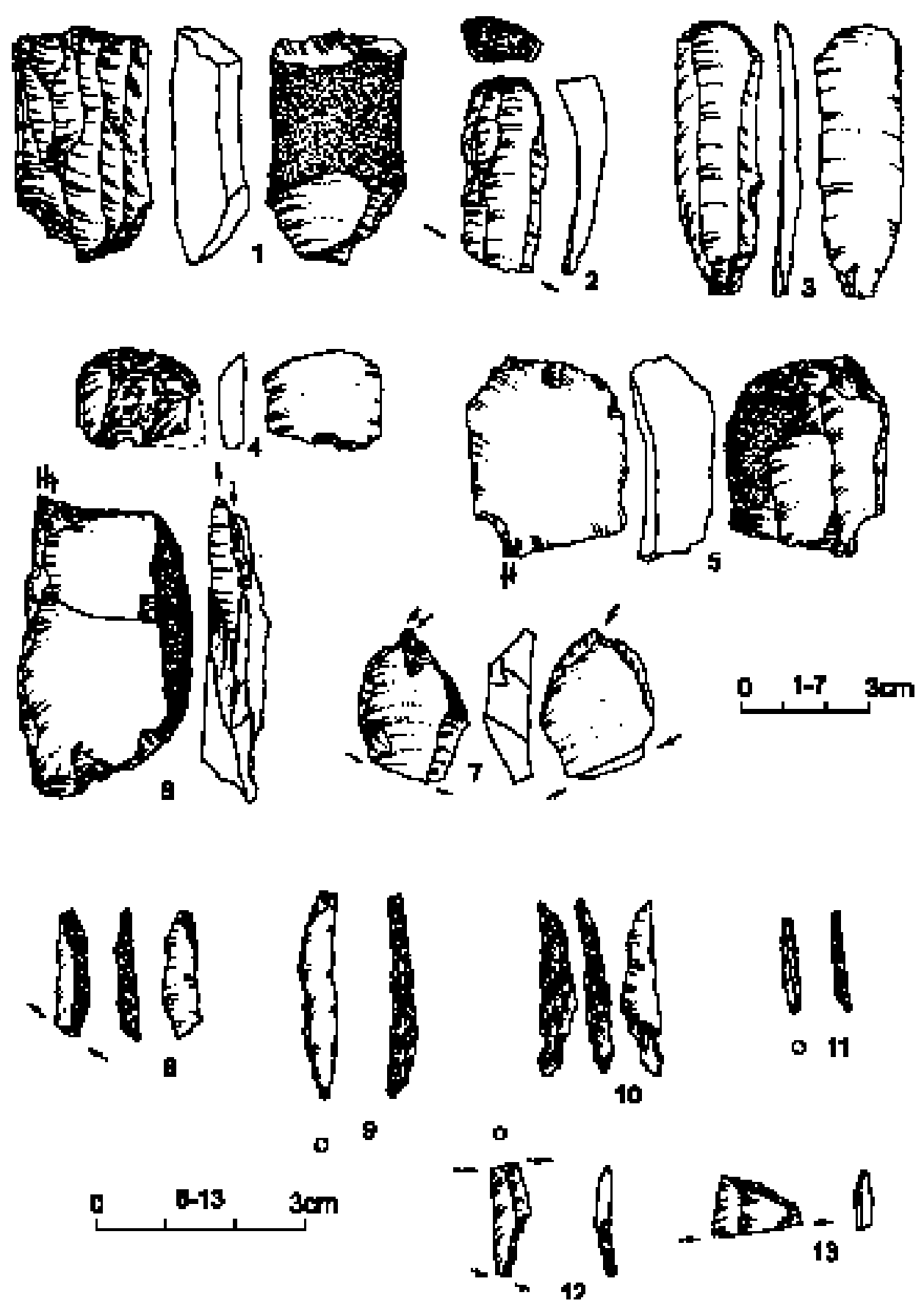

Figure 3 - Galitch I (Ukraine occidentale). Pièces à dos. D’après O.Sitnik.

Figure 3 - Galitch I (western Ukraina). Backed pieces. After O.Sitnik. 


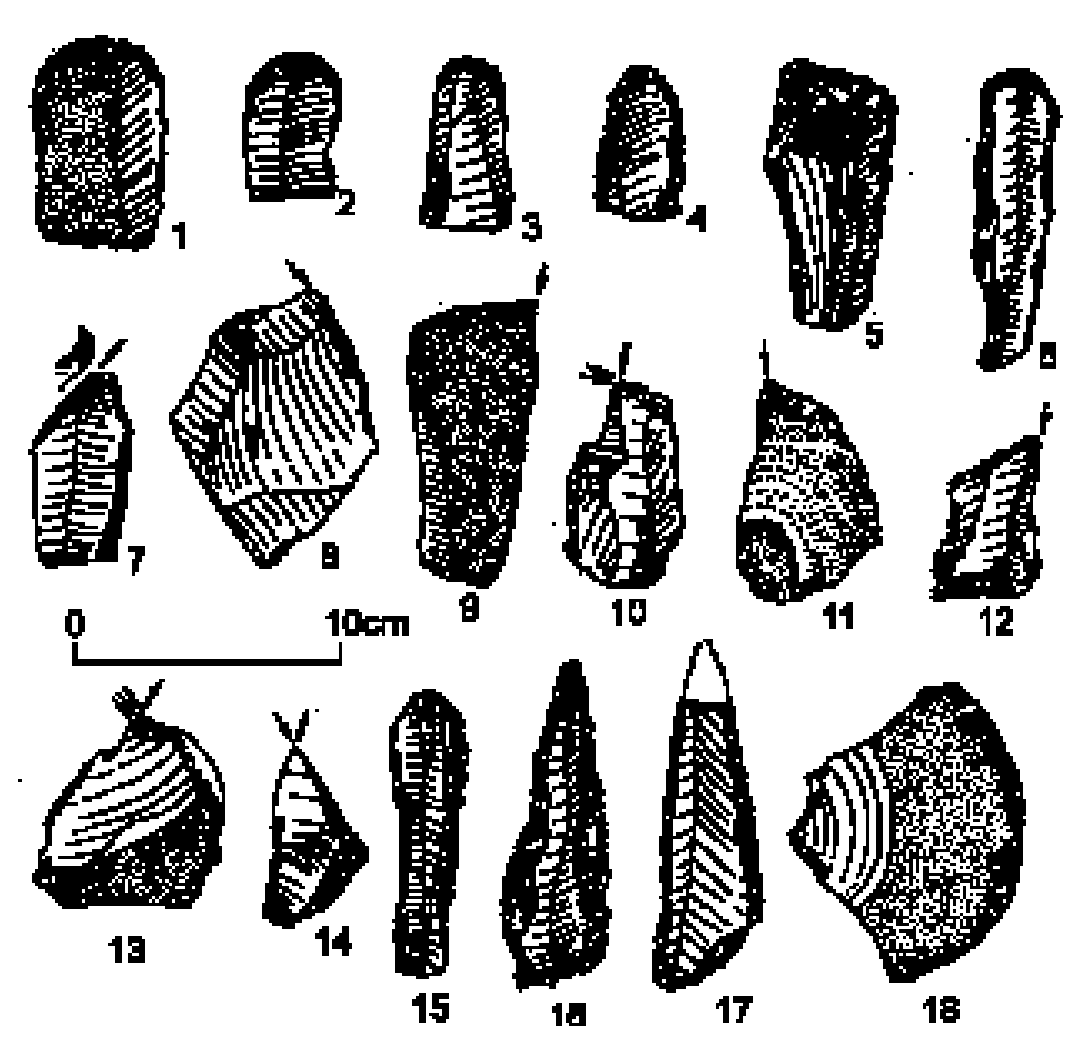

Figure 4 - Koulitchivka, niveau supérieur (Volhynie, Ukraine). Industrie lithique. D’après V.Savitch.

Figure 4 - Kulitshivka, upper level (Volhynia, Ukraina). Lithic industry. After V.Savitch.

La faune de l'Epigravettien de Lipa VI est toujours dominée par le Renne et le Mammouth. La troisième place est occupée, dans la couche 5, par le Cheval, dans la couche 3, par le Loup et dans la couche 2a par le Rhinocéros.

On évoque, depuis les années 1970, l'existence d'un faciès septentrional de l'Epigravettien de Volhynie, qu'on appelle parfois "la culture de Lipa" (Ostrovskiy et Grigoriev 1966 ; Grigoriev 1970 ; Kozlowski et Kozlowski 1975). Les recherches récentes ont bien montré (V. Sitnik 1998) que la notion de "culture de Lipa" était basée sur les ensembles volhyniens mélangés, ramassés en surface par M.I. Ostrovskiy, surtout à Mirogochtcha (Ostroivskiy et Grigoriev 1966). Les caractéristiques de cette entité sont les grattoirs à front élevé, considérés comme "aurignacoïdes", qui ne sont que des nucléus à lamelles ; des lamelles ont été également obtenues à partir des nucléus prismatiques à plan de débitage étroit, opposé à la crête postéro-centrale (Sitnik 1988, fig.1 : 1-4). Ces demiers peuvent représenter une influence du Paléolithique final. A Mirogochtcha, des outils bifaciaux, fréquemment interprétés comme des "haches", peuvent aussi être considérés comme intrusifs dans une collection de surface.

Néanmoins, certains outillages de la période du maximum du Pléniglaciaire supérieur en Volhynie sont caractérisés par la présence d'outils bifaciaux - comme des pointes foliacées, - accompagnées de grattoirs et de burins d'aspect gravettien. II s'agit du site de Griadki, récemment fouillé (V.K. Piasetskiy 2001). L'analyse palynologique des sables cryoturbés qui ont fourni cet outillage avait montré la présence de Pin et de Bouleau nain (Betula nana) qui indiquent le maximum du Pléniglaciaire.

Une association typologique semblable, datant probablement de la fin de l'Interpléniglaciaire, a été observée dans le niveau superieur de site volhynien de Jornov (Piasetskiy 1991). Notons qu'en Moldavie, la présence pérenne des pointes foliacées évoquant les pointes szélétiennes dans le contexte gravettien (mais malheureusement sans datations absolues) est bien connue de Gordinechty (Borziak 1984 ; Noiret 2003).

\section{La zone centrale}

Bases chronostratigraphiques et changements de l'environnement (tabl. 2)

Le bassin supérieur de la Vistule est une région assez variée du point de vue de l'environnement : le cours supérieur de la Vistule recoupe les plateaux calcaires et loessiques de la Pologne méridionale, cédant la place, vers le 


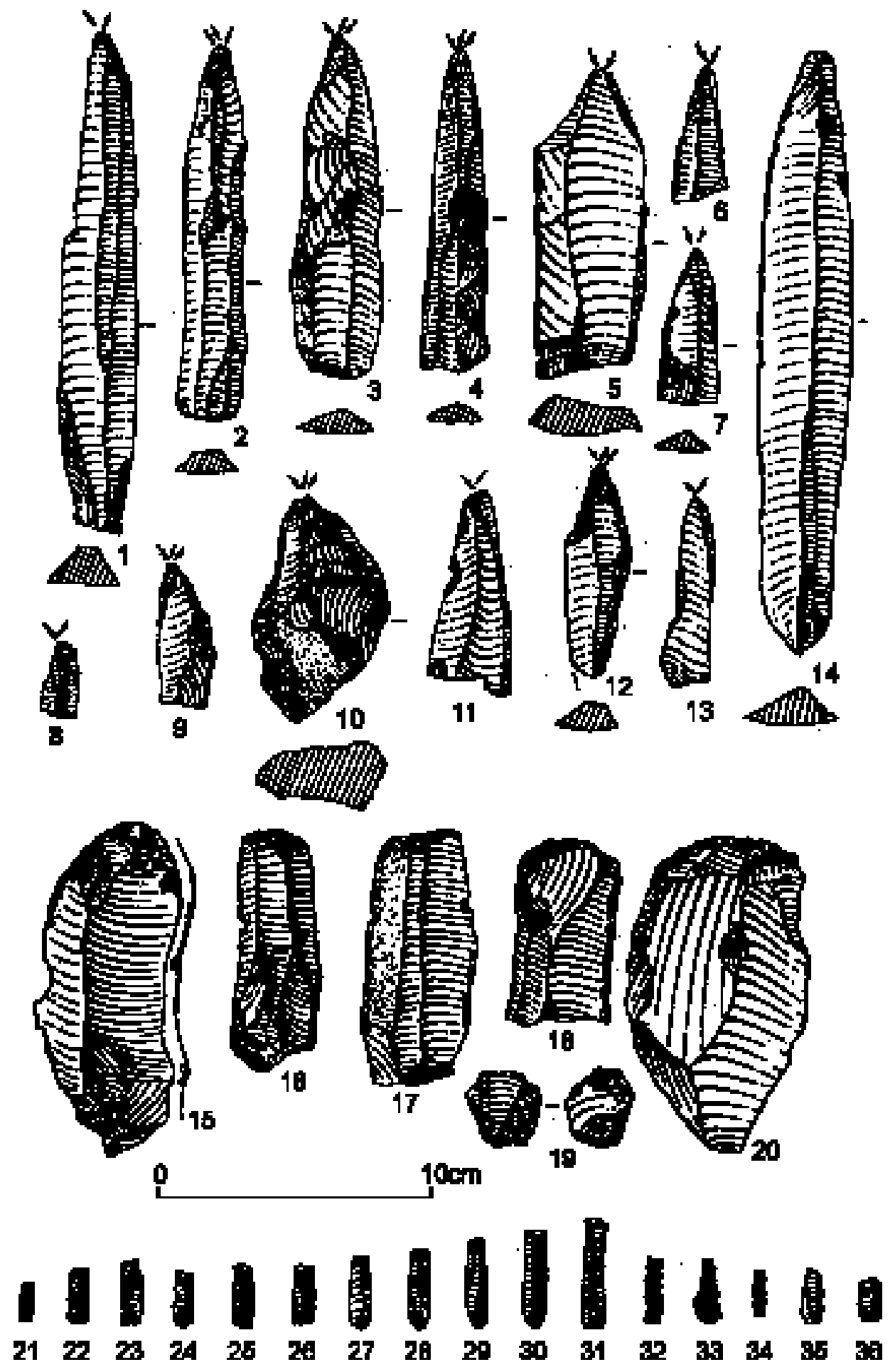

Figure 5 - Lipa VI, level 3 (Volhynie, Ukraine). Industrie lithique. D’après V.Savitch.

Figure 5 - Lipa VI, level 3 Nolhynia, Ukraina). Lithic industry. After V.Savitch. 


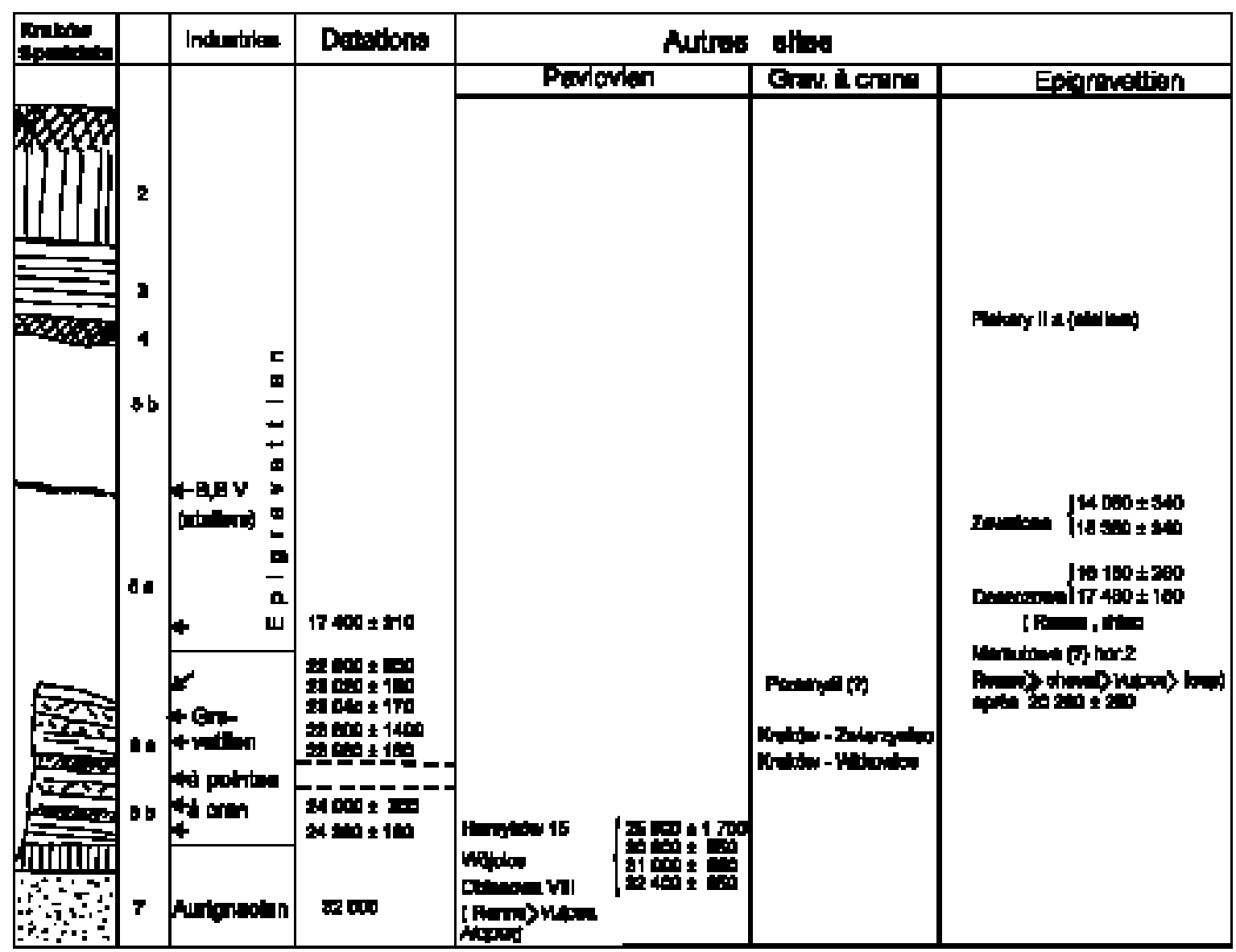

Tableau 2 - Séquence de Krakow-Spadzista comparée aux autres sites de la Pologne méridionale.

Table 2 - Krakow-Spadzista sequence compared to other south polish Gravettian sites.

sud, aux collines du Piémont des Carpates, suivi par la chaîne principale des Carpates occidentales.

Les coupes dans les loess, interprétées sur la base de la malacofaune, indiquent une stabilité des conditions climatiques durant toute la période comprise entre 30 et $20 \mathrm{Kyr}$ BP favorisant les milieux ouverts. Ce n'est que vers 23-20 Kyr BP que les ensembles de malacofaune montrent des conditions un peu plus humides et douces (Alexandrowicz 1995). Les diagrammes polliniques des dépôts tourbeux audessous de l'accumulation principale du dernier loess, indiquent, pour la vallée de la Vistule, entre 28-18 Kyr, un paysage ouvert, herbacé et très peu boisé de steppe-toundra à mammouths. Au début de la séquence (28 Kyr), les Graminées prédominent. Elles sont, par la suite, accompagnées de façon croissante par les Cypéracées. Les arbres et les buissons (surtout Pinus et Betula) ne dépassent généralement pas 10 à 15 \% (Srodon et Mamakowa 1977).

Une amélioration des conditions climatiques autour de 25 Kyr est notée, par contre, dans les grottes du Massif de Tatra, au centre des Carpates occidentales, par des formations stalagmitiques (Hercman 1991 ; Pazdur et al.1999).
Le problème essentiel, dans la zone centrale, est le début de la formation du loess récent supérieur. L'ensemble des sites de Kraków-Spadzista (Kozlowski et al. 1974 ; Kozlowski et Sobczyk 1987 ; Escutenaire et al.1999) montrent la succession d'un paléosol antérieur à $30 \mathrm{Kyr}$ BP (unité 7 ), suivi de l'unité $6 a / 6 b$ - les dépôts loessiques soliflués avec quelques arrêts de sédimentation interprété par B.Van Vliet (1992) comme équivalents de "I'horizon à langues" de Kesselt - qui ont fourni les principaux niveaux des occupations differenciées du Gravettien à pointes à cran. Ils sont surmontés par un loess lité avec gleys de toundra (unité $5 \mathrm{a}$ et $5 \mathrm{~b}$ ), contenant quelques traces d'occupation correspondant à des ateliers de transformation du silex. Cette séquence se termine par le dépôt de couverture, stratifié, avec augmentation de la composante sableuse. Elle comporte deux générations de grands coins de glace qui marquent les péjorations climatiques majeures : notamment à la limite des unités 6 et 5 (voir la coupe de site Kraków-Spadzista C1 - Kozlowski et al.1975) puis, la formation du loess sableux stratifié de l'unité 3 (cette génération a été décrite dans le site de Brzoskwinia - Van Vliet 1988). Les datations radiométriques pour Kraków-Spadzista suggèrent que le début de 

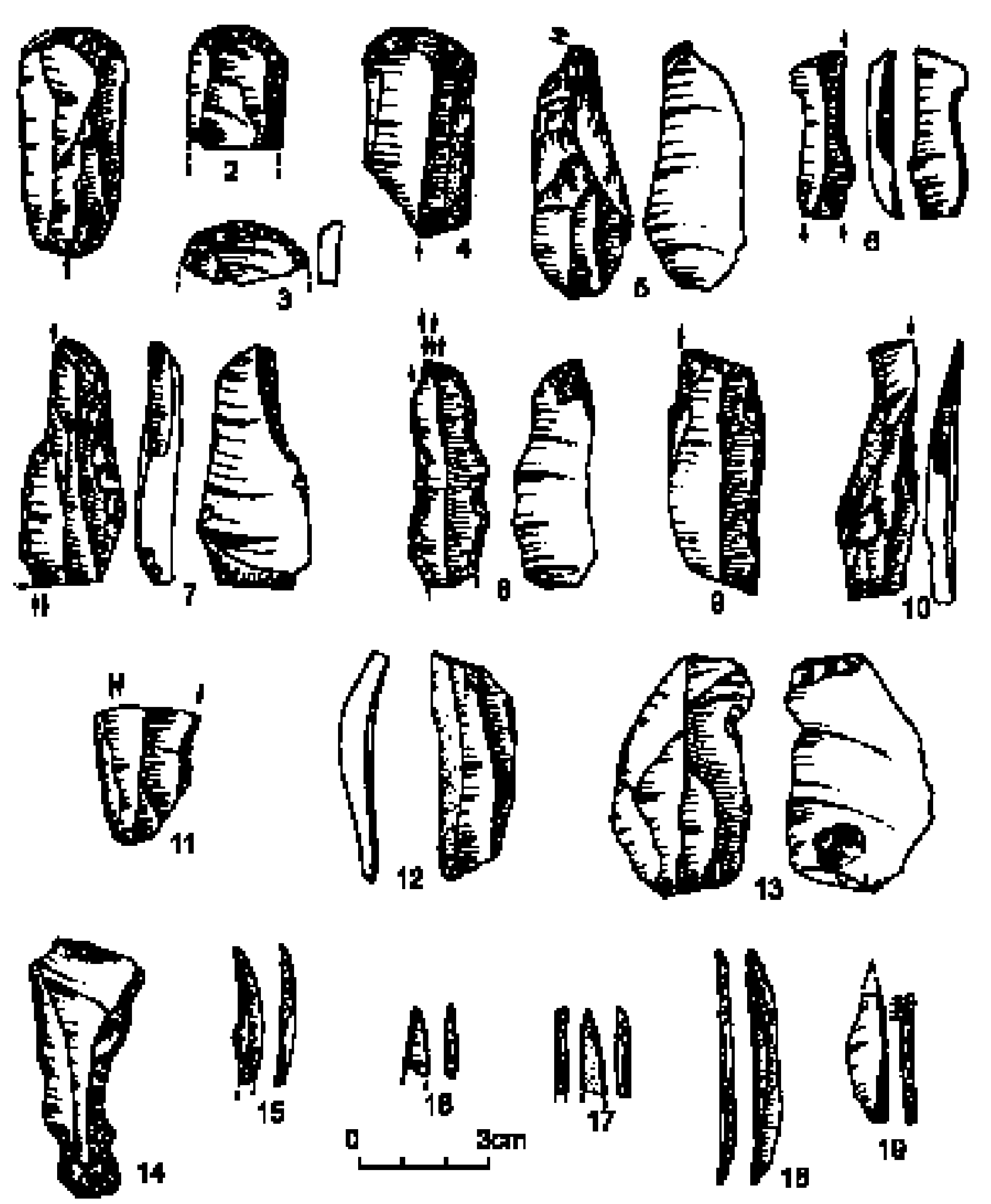

Figure 6 - Wójcice B (Silésie, Pologne). Industrie lithique. D’après B.Ginter et A. Dagnan-Ginter. Figure 6 - Wójcice B (Silesia, Poland). Lithic industry. After B.Ginter and A. Dagnan-Ginter. 
la sédimentation de l'unité loessique 5 se situerait autour de $21 \mathrm{Kyr}$, donc l'apport principal du loess serait contemporain de la période comprise entre 21 et $17 \mathrm{Kyr} \mathrm{BP}$ (Kozlowski 1996, 1998). Par contre, pour P.Haesaerts (et al. 2004) le début de l'unité 5 serait un peu plus ancien (autour de $23 \mathrm{Kyr} \mathrm{BP}$ ). Notons néanmoins que les datations TL placent le début de la sédimentation loessique dans le sud-est de la Pologne, autour de $19000 \pm 3000$ à Orzechowce et à $15000 \pm 2000$ à Radymno près de Rzeszow et à Wachock près de Kielce (Lindner 1992).

\section{Evolution du Gravettien et de l'Epigravettien}

Très peu de vestiges du Gravettien ancien et moyen - correspondant au Pavlovien morave - ont ete decouverts en Pologne. Cette situation est difficile à expliquer si l'on tient compte de l'importance (parfois jusqu'à $90 \%$ ) dans plusieurs sites classiques du Pavlovien, du silex provenant du sud de la Pologne, en particulier des moraines du bassin supérieur de l'Oder. Jusqu'à présent, seuls quelques ateliers de transformation du silex, antérieurs à la période de sédimentation du loess supérieur récent ont été découverts en Haute-Silésie (par ex. à Cyprzanów près de Racibórz-Kozlowski 1964). Le seul site de cette région à Wójcice $B$ près d'Otmuchów, dans la vallée de Nysa Klodzka - a fourni un outillage typique d'un camp de base, avec de nombreux produits de débitage sur place et des outils (environ 6600 objets), essentiellement des microlithes paragéométriques (fig.6), caractéristiques du Pavlovien (Dagnan et Ginter 1970).

La présence du Gravettien a été reconnue récemment jusqu'en Basse-Silésie grâce aux découvertes de Henrykow 15 près de Zabkowice Slaskie (Plonka et Wizniewski 2004). Ce site a fourni, dans les couches 8 et 9 , de petites concentrations de produits provenant du débitage de quelques nucléus (avec remontages) et de rares outils retouchés, tous en silex erratique provenant des moraines locales du Saalien. Les couches 8 et 9 sont probablement antérieures au début de la sédimentation du dépôt de loess récent supérieur. Parmi les outils retouchés, il n'y a que de rares lamelles à dos abattu (fig.7). II s'agit probablement de haltes de chasse dans lesquelles le travail du silex a été peu important.

Des vestiges de courts séjours des groupes gravettiens, datant d'avant $24 \mathrm{Kyr} \mathrm{BP}$, ont été découverts également dans la vallée de la Vistule près de Cracovie. A KrakówSpadzista C2, dans le niveau IV, une petite concentration avec débitage sur place et peu d'outils retouchés ont été mis au jour (Kozlowski et Sobczyk 1987) et à KrakówPrzegorzaly, un modeste atelier de transformation de silex jurassique a été découvert sous le limon de solifluxion (Kozlowski et Sobczyk 1986).

Le témoignage le plus fascinant du passage des chasseurs du Gravettien ancien provient de la couche VIII de la grotte Oblazowa près de Nowa Biala (Valde-Nowak et al.eds, 2003), située au coeur des Carpates occidentales (670 m au-dessus du niveau de la mer). II s'agit d'un outillage lithique assez pauvre (52 pièces), provenant de différentes matières premières, y compris des silex et des radiolarites importés. Parmi les produits lithiques, il y a trois nucléus (plus un pré-nucléus) en silex de Pologne centrale (silex "chocolat" et de Swieciechow) et en radiolarite (probablement slovaque ?) ainsi que des produits de débitage (à l'exception du silex "chocolat"). II n'y a que 11 outils retouchés, grattoirs et lames ainsi qu'une pièce en silex du plateau de Volhynie. L'attribution de cet assemblage au Gravettien est basée sur les pièces en os, ivoire et bois de cervidé, parmi lesquelles on trouve un coin en bois de cervidé avec décoration typique du Pavlovien (demi-cercles graves - Valde-Nowak et al. eds, 2003, phot. 15), ainsi que des perles en os et un pendentif (coquille de Conus) qui ont également des analogies avec des parures du Gravettien morave. La couche VIII a également foumi un objet cérémonial en forme de boomerang (ValdeNowak 2000). La position de cet objet à l'intérieur d'un cercle de gros galets de rivière transportés au fond de la grotte, suggère un lieu de cérémonie (fig.8). La présence, dans ce cercle, de phalanges humaines pourrait être liée à des cérémonies d'initiation, comme en témoignent les "mains mutilées" dans l'art gravettien franco-cantabrique (Valde-Nowak 2003).

L'occupation du niveau VIII d'Oblazowa doit être rapportée au tout début du Pavlovien, autour de $30 \mathrm{Kyr}$ BP, témoignant du franchissement des Carpates par les populations du Gravettien ancien et la pénétration, non seulement d'une large zone du bassin supérieur de la Vistule, mais aussi du Plateau de Volhynie. De plus, cette occupation suggère l'implication, dans ces expéditions saisonnières de chasse surtout estivale, de personnes responsables des rites et des cérémonies.

Si le Gravettien ancien et moyen n'est connu, dans le sud de la Pologne (à l'exception du site de Wojcice), que par des haltes de chasse et des ateliers de transformation de silex, le Gravettien récent - horizon à pointes à cran - est connu par d'importants camps de base, lieux d'abattage et de dépeçage des mammouths, de grands ateliers de trasfomation de silex et, bien sûr par des camps éphémères. Le grand complexe de sites, datant de la période comprise entre 24 et $23 \mathrm{Kyr}$ BP est connu à KrakówSpadzista (Kozlowski 2003, 2004 ; Kozlowski et MontetWhite 2001 ; Wojtal et Sobczyk 2005).

Le tableau 3 montre la différenciation chronologique et fonctionelle des occupations gravettiennes dans cet ensemble de sites. Les differents loci ont fourni des outillages caractérisés par la présence de pointes à cran, de pièces à dos, de couteaux de Kostienki et de très nombreux burins et de quelques grattoirs (fig. 9). L'utilisation des pointes à dos et à cran comme pointes de projectiles est confirmée par des fractures d'impact, bien que l'analyse tracéologique et leur mode d'emmanchement montrent que ces outils ont également été utilisés comme des couteaux à couper la viande. L'amas des ossements de mammouths - principalement lieu de boucherie - a fourni les restes d'au moins 86 mamouths (Wojtal, Sobczyk 2005), mais l'aire d'accumulation est probablement deux fois plus importante que la zone fouillée. Par contre, les lieux de campement n'ont fourni que 

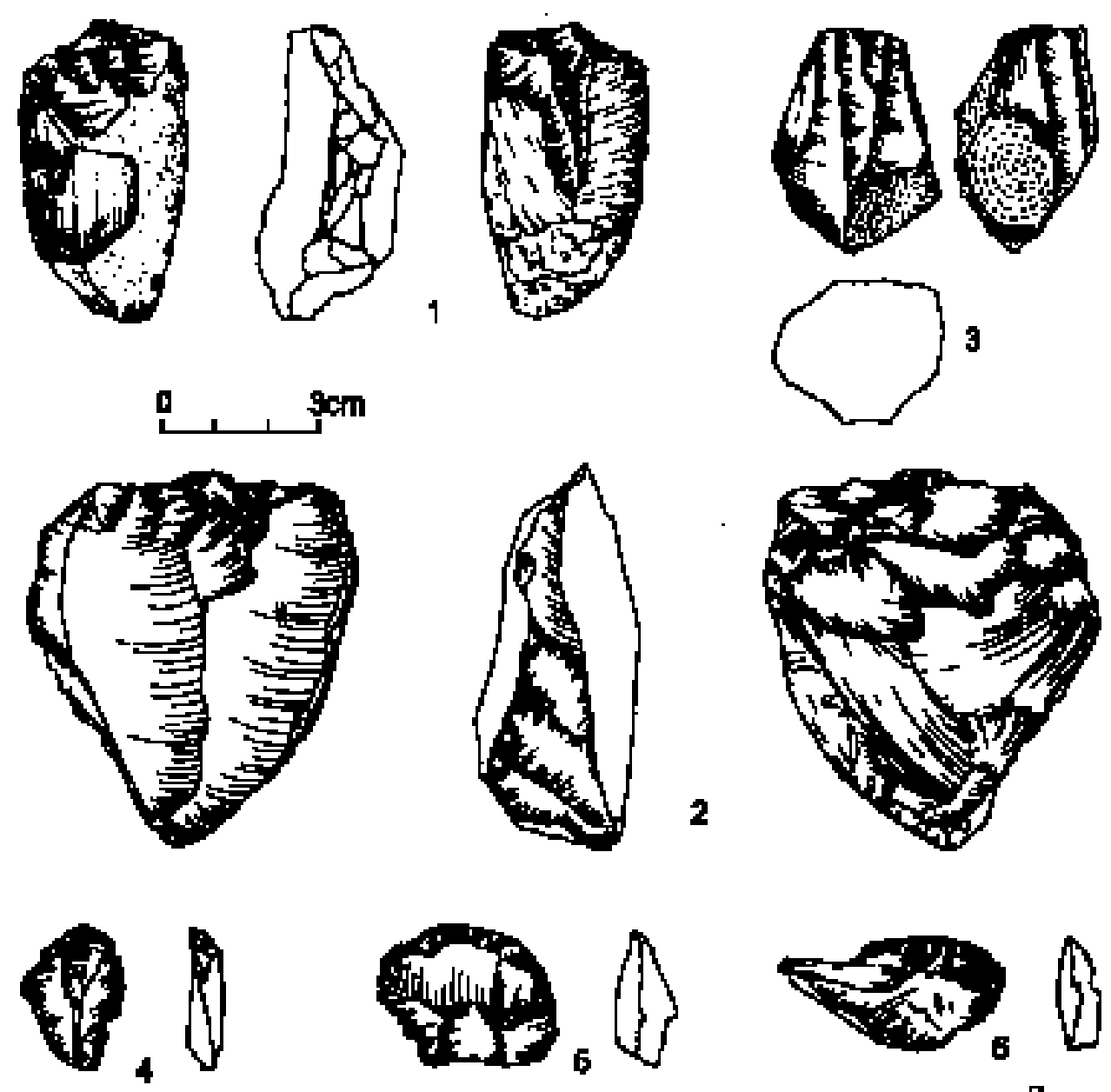

2
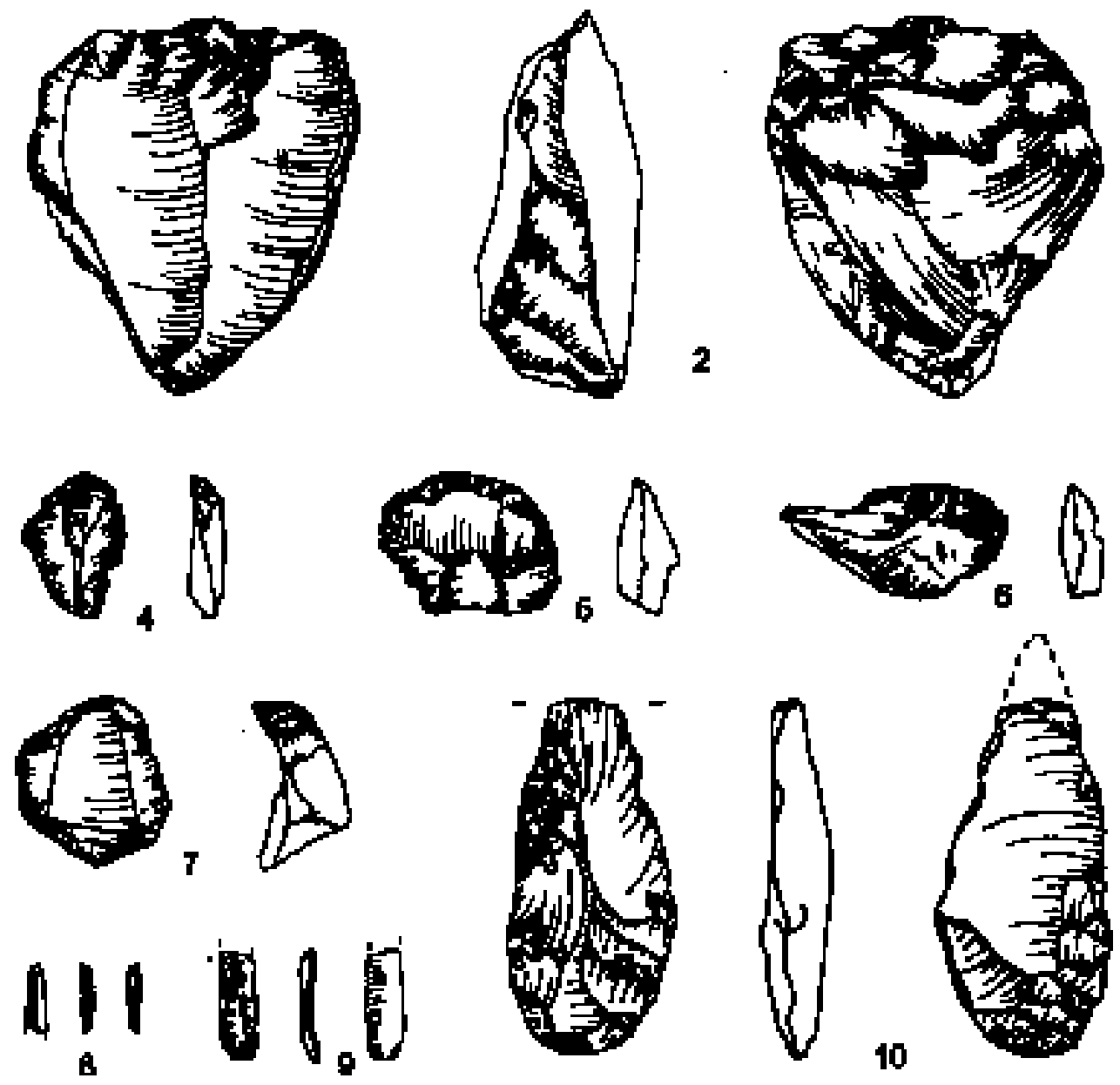

Figure 7 - Henryków 15 (Silesie, Pologne). Industrie lithique. D’après T.Plonka et A.Wisniewski.

Figure 7 - Henryków 15 (Silesia, Poland). Lithic industry. After T.Plonka et A.Wisniewski. 


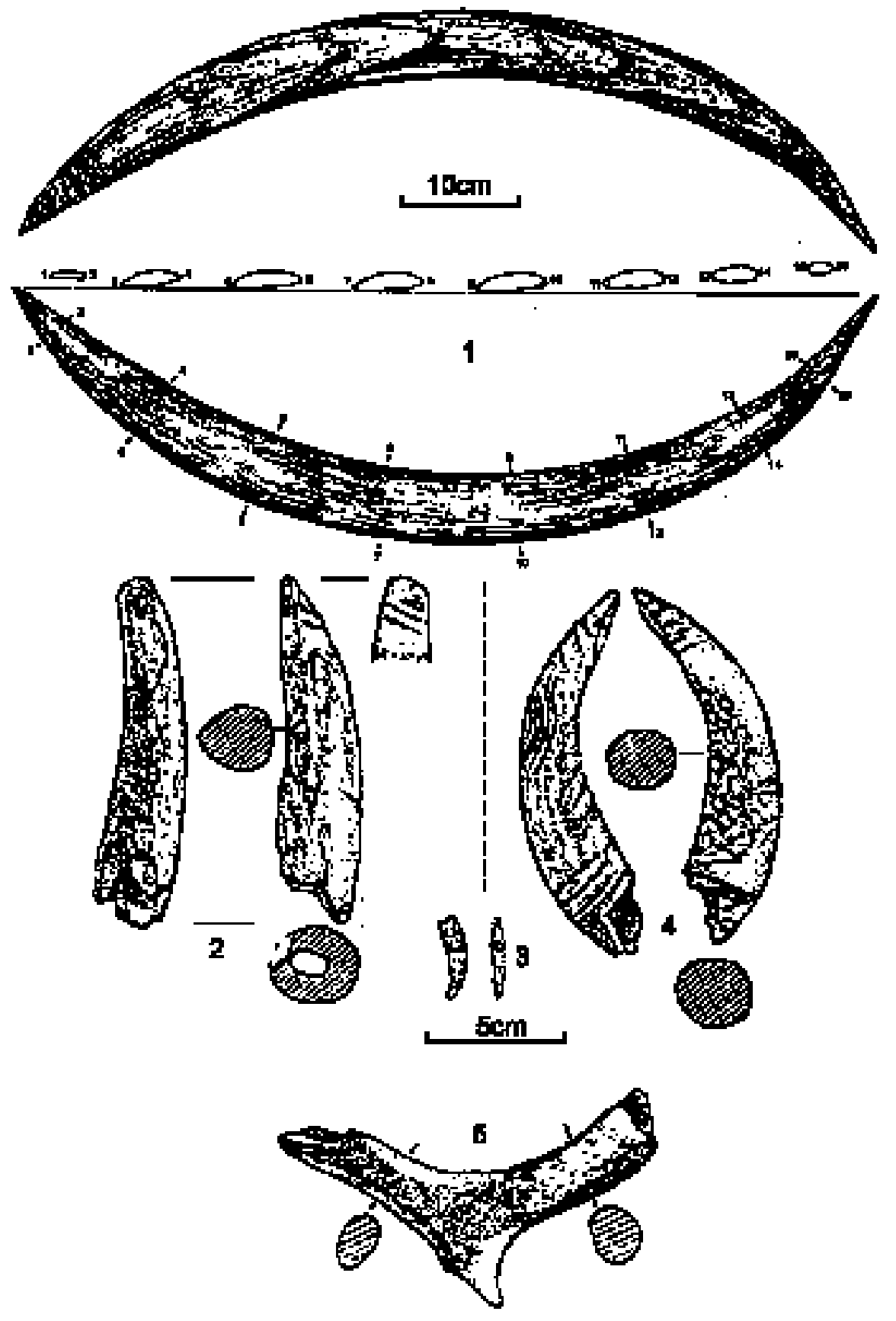

Figure 8 - Grotte Oblazowa (Carpates, Pologne). Plan du niveau VIII. D’après P.Valde-Nowak.

Figure 8 - Oblazowa Cave (Carpathians, Poland). Scatter-pattemof artefacts and pebbles in level VIII. After P.Valde-Nowak. 


\begin{tabular}{|c|c|c|c|c|}
\hline 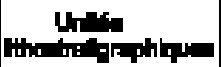 & sam ar bouthore & Camper of best & Campe indmere & Anlalm: \\
\hline Lotistoptinur & & & 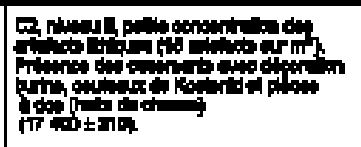 & \\
\hline EH & & & 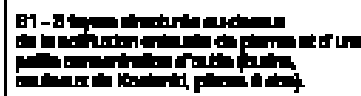 & \\
\hline \multirow{8}{*}{ 0. } & & 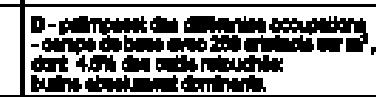 & & \\
\hline & & & 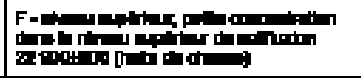 & \\
\hline & & & t & 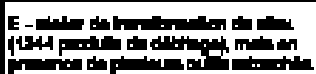 \\
\hline & 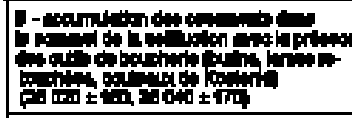 & & & \\
\hline & & 4 & 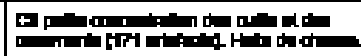 & \\
\hline & & 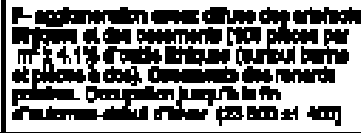 & & \\
\hline & & 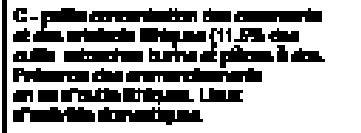 & & \\
\hline & | L & & & \\
\hline Pallonel| & & & & \\
\hline \multirow{3}{*}{ th } & 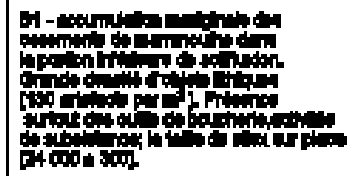 & & & \multirow{3}{*}{ 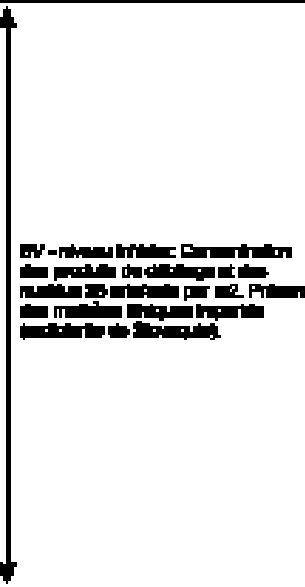 } \\
\hline & & 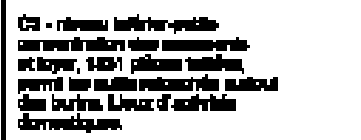 & & \\
\hline & & 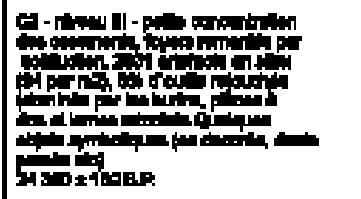 & & \\
\hline$\theta$ & 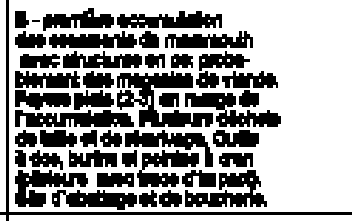 & & & \\
\hline$a$ & & 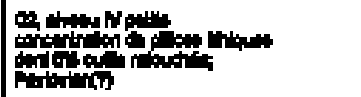 & 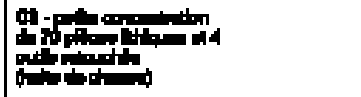 & \\
\hline
\end{tabular}

Tableau 3 - Séquence des différents types d'occupations gravettiennes de Krakow-Spadzista.

Table 3 - Sequence of différent occupation phases and activities at the Gravettian site of Krakow-Spadzista. 


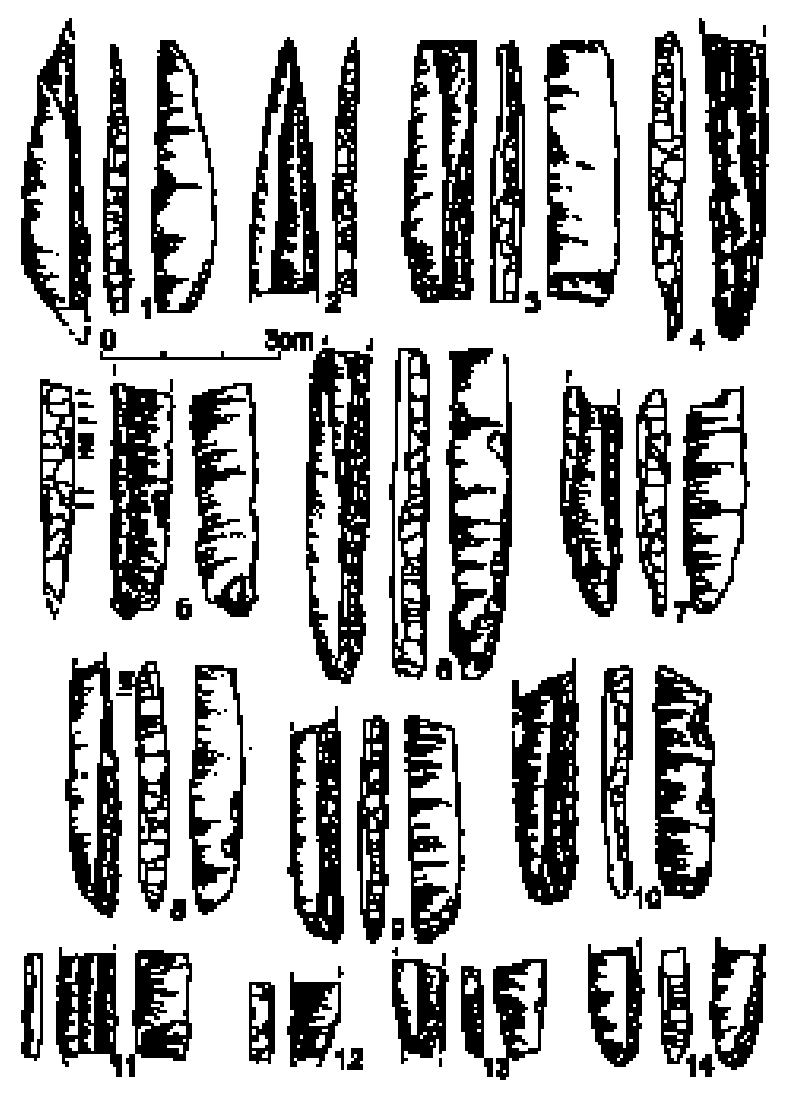

$\mathbf{A}$
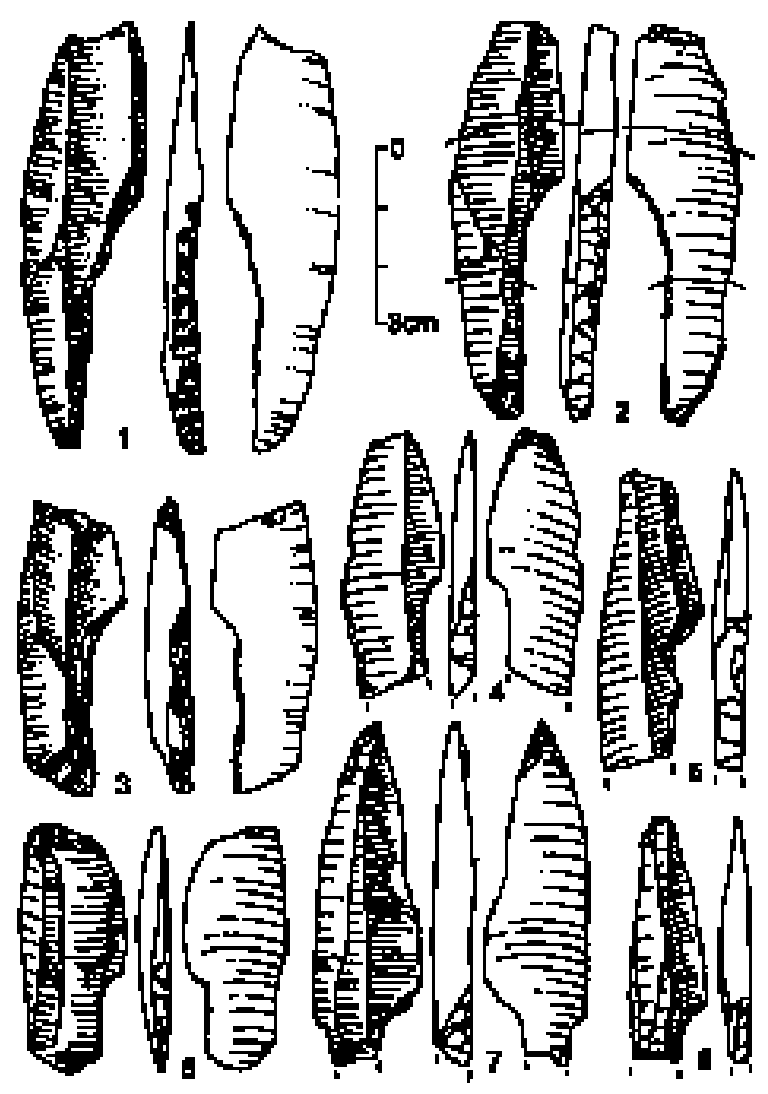

$\mathbf{B}$

Figure 9 - Kraków-Spadzista B (Pologne). Industrie lithique du niveau 6.

Figure 9 - Kraków-Spadzista B (Poland) Lithic industry from layer 6.

les vestiges de un à trois mammouths, accompagnés de quelques ossements de Renne, Cheval, Rhinocéros, Ours et Loup. Notons aussi, dans le locus F, la présence de Renards polaires, dont l'étude des dents suggère une prolongation de séjour, en principe de la fin printemps/été jusqu'à la fin de l'automre/début d'hiver (West 1996).

La fin de l'occupation de Krakow-Spadzista correspond à la période d'accumulation du dernier loess avec quelques gley de toundra (couche 5) ; dans cette phase, les formes diagnostiques du Gravettien récent - pointes à cran et couteaux de Kostienki - disparaissent. Les sites de cette période ne sont représentés que par des haltes de chasse (par exemple Kraków-Spadzista C2 niveau II et I - Kozlowski et Sobczyk 1987) et des ateliers de transformation de silex (locus B-V - Drobniewicz et al. 1976). Dans cette région, il s'agit de la fin de la pénétration systématique du bassin supérieur de la Vistule à partir du centre ouest de la Slovaquie, dans la vallée de Vah par les groupes du Gravettien à pointes à cran (Kaminska et Kozlowski 2002) ; ces groupes ont abandonné l'Europe centrale au moment du maximum du Pléniglaciaire pour continuer leurs stratégies de subsistance liées à la chasse au mammouth en
Europe de l'est (Kozlowski 1986 et Soffer 1993).

Le caractère des sites épigravettiens post-pléniglaciaires, en Pologne méridionale, témoigne plutôt en faveur de courtes pénétrations, surtout liées à l'approvisionnement en matières premières lithiques. Cette hypothèse contraste un peu avec ce qui a été observé dans la grotte Mamutowa à Wierzchowie (Plateau Jurassique CracovieCzestochowa) qui a fourni, dans le loess postérieur à 20 Kyr BP (couche 2/2g - Kozlowski et Kozlowski 1996, pl. 46) une abondante industrie épigravettienne avec une technologie de lames très régulières obtenues à partir de nucléus bipolaires, évoquant certains assemblages de Volhynie (par exemple de la couche 2a de Lipa VI) et du bassin du Dniester moyen (Molodovien récent), ainsi que la composition typologique comportant de nombreux burins et de rares lamelles à dos (fig.10). La faune, assez abondante (458 éléments) de cet assemblage, a fourni les restes d'Ours (cMNI-9) et de Lapin (cMNI-10), suivis du Renne (3), du Cheval (1) et de plusieurs autres animaux de milieux ouverts (Rhinocéros, Bison, Ovibos, Saïga). Bien que pour les lapins et les rennes, il faille supposer qu'ils ont été dépecés dans la grotte, pour les autres, il est difficile de savoir s'il s'agit de l'apport, dans la grotte, de restes d'ani- 


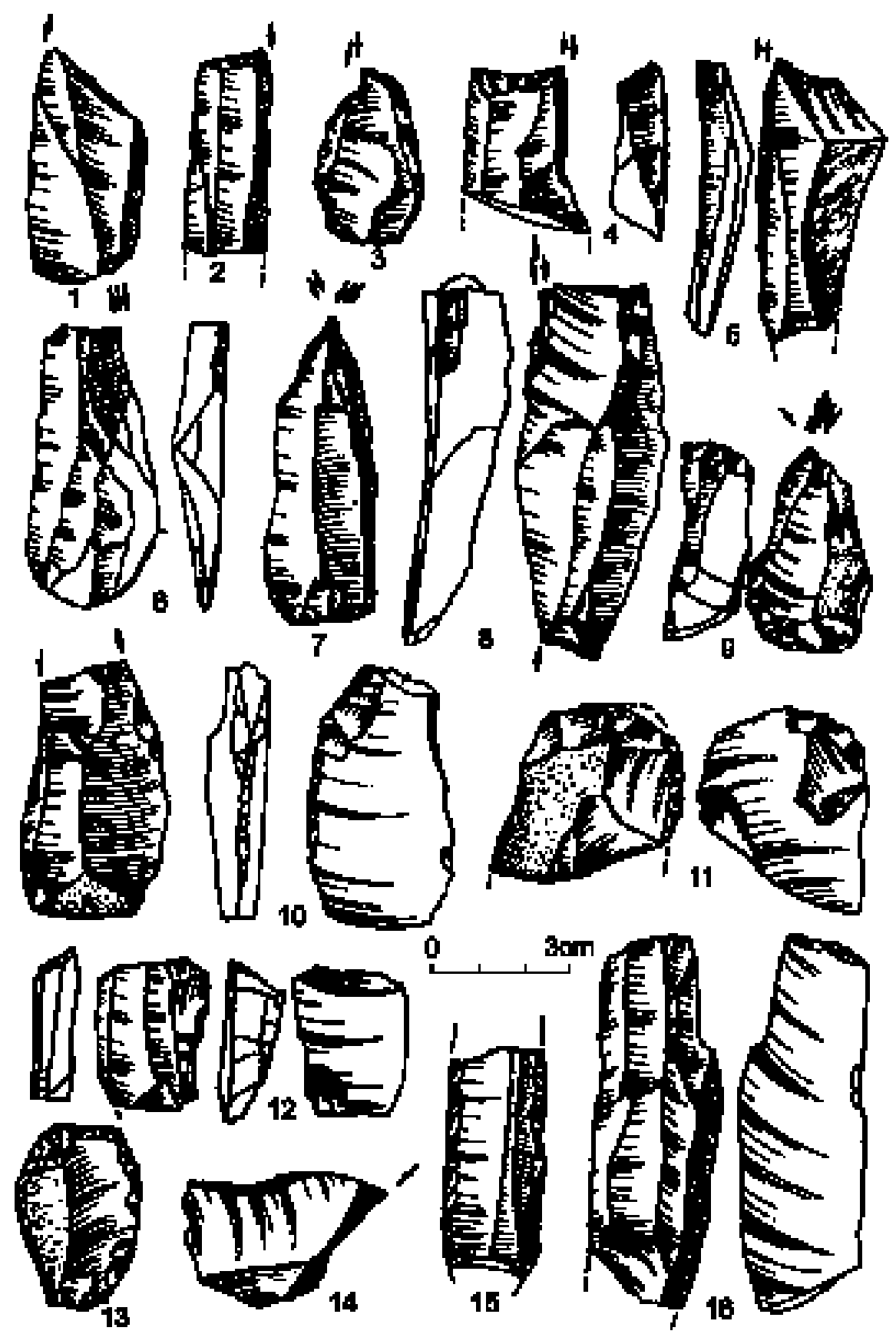

Figure 10 - Grotte Mamutowa (Pologne). Industrie lithique de la couche 2/2g.

Figure 10 - Mamutowa Cave (Poland). Lithic industry from layer 2/2g. 

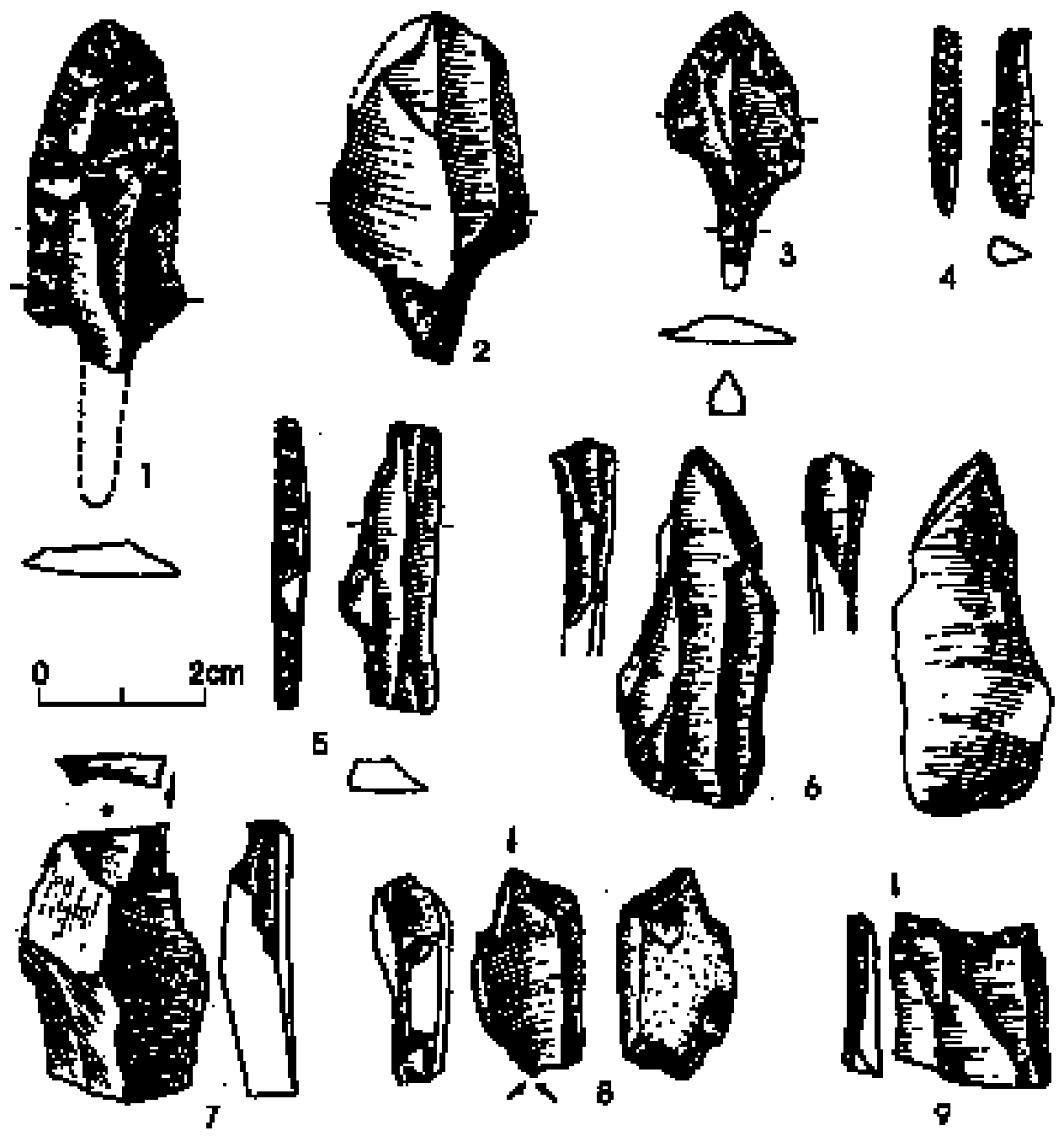

5
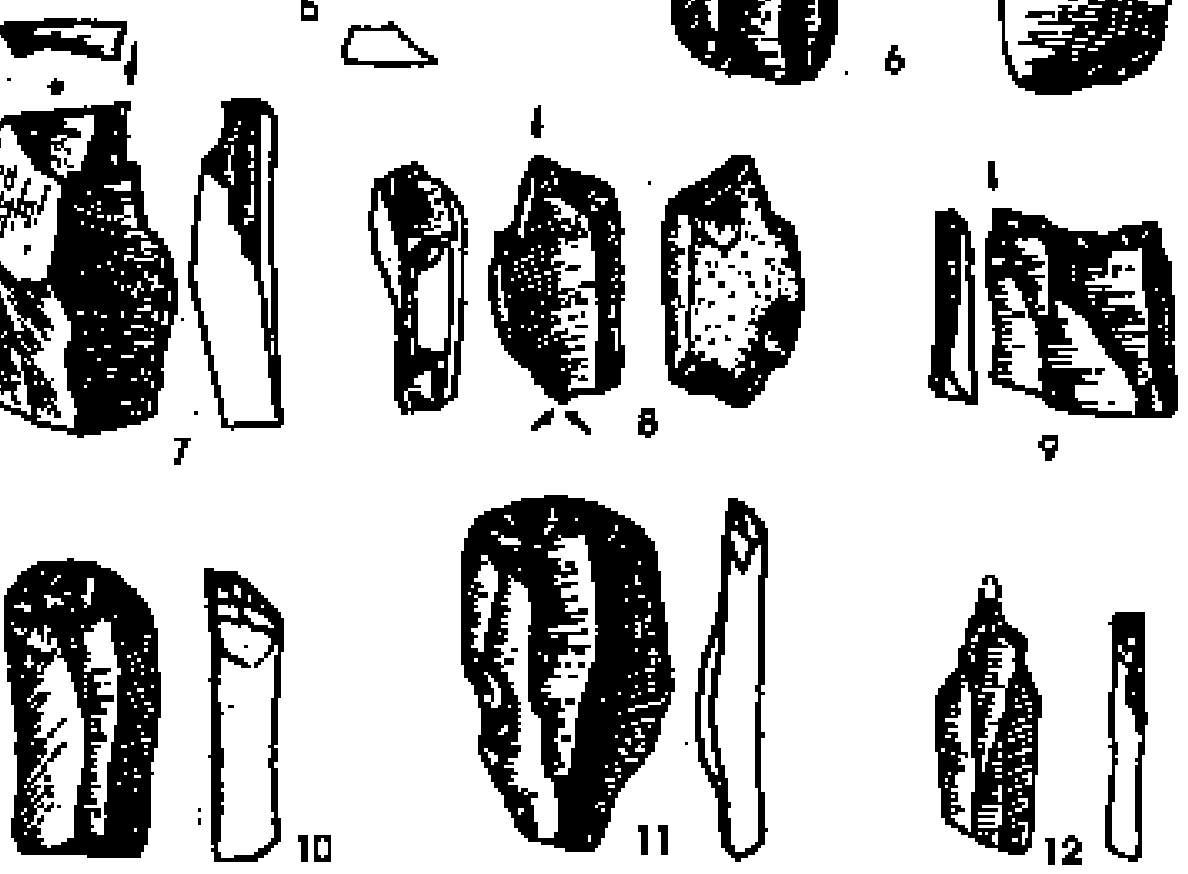

Figure 11 - Bilzingsleben (Thuringe, Allemagne). Pointes pédonculées. D’après D. Mania.

Figure 11 - Bilzingsleben (Thuringia, Germany). Tanged points. After D. Mania. 
maux désarticulés par l'homme ou par les camivores (Pean et Wojtal 2003). Le Mammouth n'est connu que par des fragments d'ivoire.

Les autres habitats épigravettiens fouillés dans les grottes Deszczowa I and II, dans la partie septentrionale du Plateau Jurassique de Kraków-Czestochowa, ressemblent à des haltes de chasse. Par exemple, le niveau 3 de Deszczowa I avec une datation $17480 \pm 150$ (Cyrek 1999) n'a fourni que 22 objets dont huit outils retouchés : burins, grattoirs et lames (certains avec traces d'aménagements secondaires et tertiaires). A ces outils, il faut ajouter trois poinçons en os et deux ébauches de perles en bois de cervidé et en os. Dans la faune, le Renne et le Lapin sont les plus fréquents: les carcasses complètes de renne ont été apportées dans la grotte ; cette chasse a eu lieu en automne (Pean et Wojtal 2003). L'utilisation de l'Ours des cavernes, probablement pour la fourrure, est confirmée par les traces de dépeçage sur les phalanges et les métapodes. Notons aussi la présence de l'Antilope Saïga, dont la carcasse a été dépecée à l'extérieur de la grotte. Le site Deszczowa II est un autre exemple de bref séjour d'un groupe épigravettien, qui n'a pas laissé de mobilier archéologique. La présence de l'hom- me est attestée ici par les ossements de cinq rennes tués en été et dépecés dans la cavité, ainsi que ceux de trois lapins et de deux chevaux (Pean et Wojtal 2003).

Les sites épigravettiens mentionnés ici confirment que les brèves visites saisonnières de groupes humains ont eu lieu au nord des Carpates immédiatement après le maximum glaciaire, surtout pour la chasse, pendant la période été/automne. Ces visites ont continué dans le Tardiglaciaire jusqu'à la période des premières intrusions magdaléniennes autour de 15-14 Kyr BP (Kozlowski 1999).

\section{La zone occidentale}

Les habitats gravettiens sont trés peu nombreux dans la zone occidentale. Dans l'interfleuve de l'Elbe et de la Saale, nous ne connaissons pratiquement que deux sites gravettiens à Bilzingsleben près de Weimar et à Gera-Zoitsberg. Ces deux sites n'ont rien de commun avec le Pavlovien ou le Gravettien récent à pointes à cran (Mania 1981).

Quelques objets lithiques trouvés à Bilzingsleben représentent le Gravettien ancien de type occidental, notam-

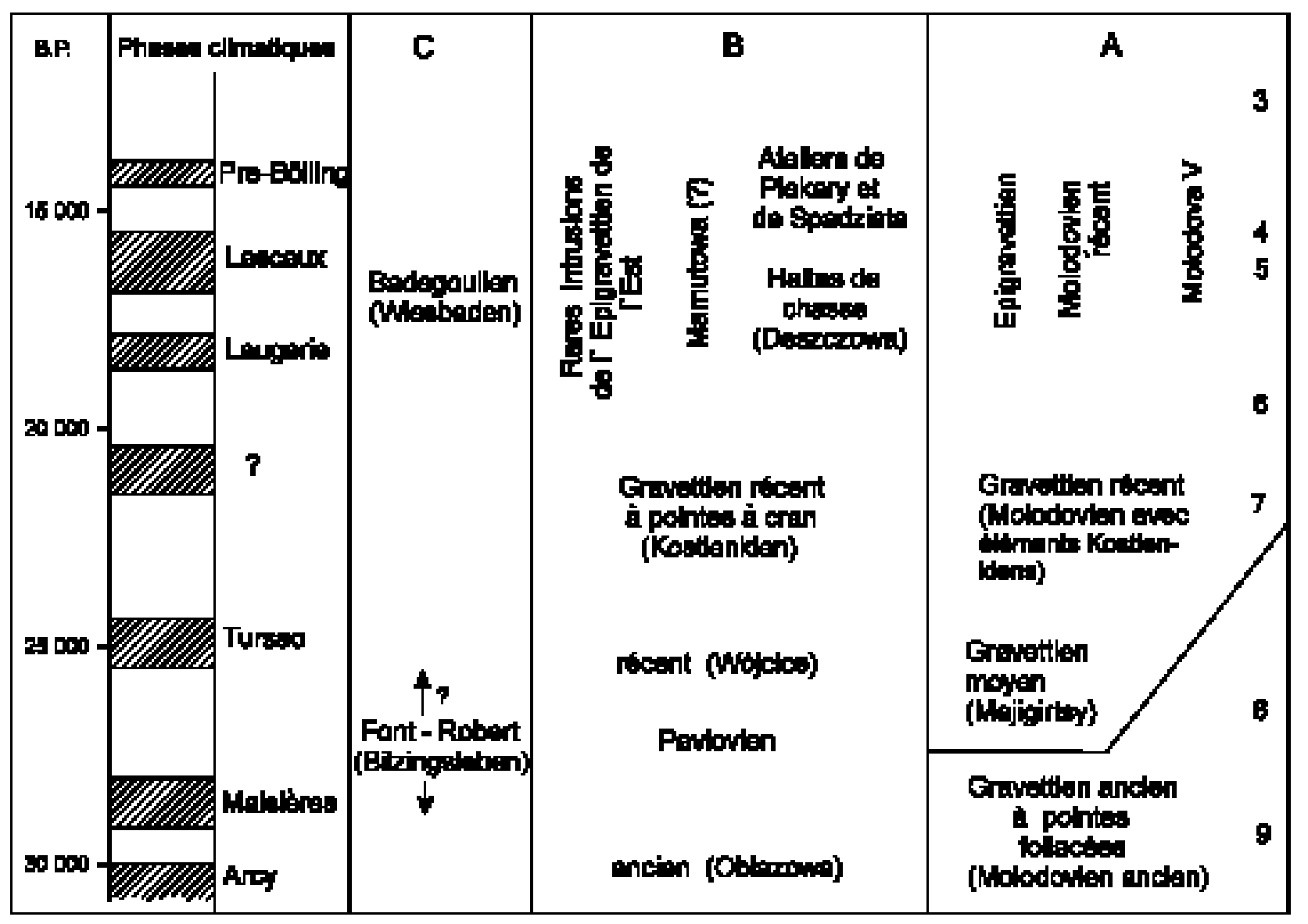

Tableau 4 - Synthèse de l'évolution du Gravettien/Epigravettien dans les trois zones géographiques étudiées.

Table 4 - General evolution of the Gravettien/Epigravettien in three discussed zones. 
ment le Fontirobertien, caractérisé par la présence de pointes pédonculées (fig.11).

Le site de Gera-Zoitzberg a été attribué par R.Feustel (1965) au Magdalénien ancien. Après la découverte d'un assemblage à Wiesbaden-Ingstadt datant du maximum du Pléniglaciaire (âge moyen 19 Kyr BP) et qui présente des caractères technologiques proches du Badegoulien (Terberger et Street 2002), on peut se demander si, dans le cas de Gera-Zoitzberg, il ne s'agit pas également de cette même industrie d'origine occidentale.

\section{CONCLUSIONS (tabl. 4)}

Nous avons essayé, dans cet article, de démontrer que la zone située au nord des Carpates et des Sudètes n'a été que temporairement fréquentée par les chasseurs gravettiens en provenance de centres pavloviens du bassin danubien. Les preuves d'existence de liens systématiques entre les deux versants des Carpates occidentales n'apparaissent que dans la période du Gravettien récent à pointes à cran, avant l'émigration des chasseurs du Gravettien récent vers la Plaine russe, où ils ont fondé la culture de Kostenki-Avdeevo.

Si la zone centrale de la Vistule et de l'Oder a fonctionné comme un système lié au bassin carpatique et la zone orientale au bassin du Dniester moyen, par contre, la zone occidentale ne fut que très rarement occupée par les chasseurs gravettiens occidentaux.

\section{BIBLIOGRAPHIE}

ALEXANDROWICZ S.W. 1995 - Malacofauna of the Vistulian loess in the Krakow region (S.Poland). Annales Universitatis Mariae Curie-Sklodowska, L/, 1-28.

BOGOUTSKIY A.B. 1986 - Antropogenovyie pokrovnyie otlojenia Volyno-Podolii. In: Antropogenvyie otlojenia Ukrainy, Kiev, Naoukova Doumka, 121-132.

BORZIAK I.A. 1984 - Verkhnepaleoliticheskaya stoyanka Gordineshty I w Poputie.Kishinev:Shtintsa.

BORZIAK I.A., GRIGORIEVA G.V., KETRAROU N.A. 1981 Poselenia drevniekamiennogo veka na severo-zapade Moldavii. Kishinev:Shtintsa.

CHERNYSH A.P. 1959 - Paleolit sredniego Podniestrovia. Moskva:Naouka.

CHERNYSH A.P. 1961 - Molodova V. Kiev, Naukova Doumka.

CHERNYSH A.P., 1973 - Paleolit i mezolit Pridniestrovia. Moskva:Naouka.

CHERNYSH A.P. 1987 - Etalonnaya mnogosloynaya stoyanka Molodova V. Moskva:Naouka.
CYREK K. 1999 - Menschliche Penetration der Hohlen im mittleren Teil der Krakowsko-Cestochowska-Hochenebene zwischen 1.und dem 11.Jahrtausend (vom Interstadial Lascaux bis zum Interstadial Allerod). Folia Quaternaria, 70, 269-288.

DAGNAN-GINTER A., GINTER B. 1970 - Wyniki badan wytkopaliskowych przeprowadzonych na stanowisku gornopaleolitycznym w Wojcicach pow.Grodkow. Sprawozdania Archeologiczne, 22, 31-36.

DJINDJIAN F. 2002 - Ruptures et continuités dans les industries du maximum glaciaire en Europe centrale et orientale : la question de l'Epigravettien. In : Trends in the Evolution of the East European Palaeolithic, Kostenki in the context of the Palaeolithic of Eurasia. Saint-Pétersbourg, 250-255.

DROBNIEWICZ B., KOZLOWSKI J.K., SACHSE-KOZLOWSKA E. 1976 - Studia nad technika obróbki kamienia w gómym paleolicie. Pracownia krzemieniarska na stanowisku Kraków ul.Spadzista (B). Archeologia Polski, 21, 39-83.

ESCUTENAIRE C., KOZLOWSKI J.K., SITLIVY V., SOBCZYK K. 1999 - Les chasseurs de mammouths de la vallée de la Vistule. B ruxelles: Musées Royaux d'art et d'Histoire. Feustel R., 1965. Das Aurignacien von Zoitzberg bei Gera. Alt-Thuringen, 8, 15-39

GOZHIK P., SHELKOPLYAS V., KHRISTOFOROVA T. 1995 - Development stages of loessial and glacial formations in Ukraine. Annales Universitatis M. CurieSklodowska, 50, 65-74.

GRIGORIEV G.P. 1970. Verkhniy paleolit. In: Kamennyi viek na territorii SSSR, Moskva, 43-61.

HEASAERTS P., BORZIAK I., CHIRICA V., DAMBLON F., KOULAVSKA L. 2004 - Cadre stratigraphique et chronologique du Gravettien en Europe centrale. In: The Gravettian along the Danube. Brno, 33-56.

HERCMAN H. 1991 - Rekonstrukcja elementów srodowiska geologicznego Tatr Zachodnich na podstawie datowania izotopowego nacieków jaskiniowych. Zeszyty Naukowe Politechniki Slaskiej 66, Geochronometria 8.

IVANOVA I.K., TSETLIN S.M. 1987 - Mnogosloynaya paleolitcheskaya stoyanka Molodova V, Moskva, Naouka.

KAMINSKA L., KOZLOWSKI J.K. 2002 - Gravettian settlement on the south and north side of the Western Carpathians. In : Starsza $i$ srodkowa epoka kamienia w Karpatach Polskich, Krosno, 35-58.

KLAPCHUK N. 1983 - Novyie dannyie o paleolitie i mezolitie Prikarpatia. Sovietskaya Arkheologia, 4, 103-118.

KOULAKOVSKAYA L. 1989 - Nowyie dannyie o paleolitie Podnestrovia. In : Kamiennyi viek: pamiatniki, metodika, problemy.Kiev:Naouka, 50-61. 
KOULAKOVSKAYA L., OTTE M.1999 - Mejigirzi. Préhistoire Européenne, 13, 149-166.

KOZLOWSKI J.K.1964 - Paleolit na Górnym Slasku. Wroclaw:Ossolineum.

KOZLOWSKI J.K. 1986 - The Gravettian in Central and Eastern Europe.Advances in World Prehistory 5,131-200.

KOZLOWSKI J.K. 1996 - The Danubian Gravettian as seen from the Northern Perspective (dans) Palaeolithic in the Middle Danube Region, Brno:Archeologicky Ustav AV CR, 11-22.

KOZLOWSKI J.K. 1999 - Les origines de la recolonisation de la partie septentrionale de l'Europe Centrale apres le Pléniglaciaire. Folia Quatemaria, 70, 317-332.

KOZLOWSKI J.K. 2003 - Mammoth bone accumulations and dwelling structures: discussing some arguments around Krakow-Spadzista B site. In: Percieved Landscapes and Built Environments, BAR Intemational Series, 122, 59-64.

KOZLOWSKI J.K., 2004 - Le site Gravettien de Cracovie rue Spadzista (Pologne méridionale) et ses amas d'ossements de mammouth. Dossiers d'Archeologie, 291,60-63.

KOZLOWSKI J.K., KOZLOWSKI S.K. 1975 - Pradzieje Europy od XL do V tysiaclecia. Warszawa PWN.

KOZLOWSKI J.K., KOZLOWSKI S.K. 1996 - Le Paléolithique en Pologne. Grenoble : Millon.

KOZLOWSKI J.K., MONTET-WHITE A. 2001 - The huntingbutchering tool-kit from Krakow-Spadzista: a Gravettian site in southern Poland. In : Proceedings of the Interational Conference on mammoth site studies, Kansas, 109-116.

KOZLOWSKI J.K., SOBCZYK K. 1987 - The Upper Palaeolithic site Krakow-Spadzista street C2. Prace Archeologiczne,42, 7-68.

KOZLOWSKI J.K., KUBIAK H., SACHSE-KOZLOWSKA E., VAN VLIET B., ZAKRZEWSKA G. 1974 - Upper Palaeolithic site with dwelling of mammoth bones Cracow-Spadzista street (B). Folia Quatemaria, 44.

KOZLOWSKI J.K., VAN VLIET B., KRAMARZ K., DROBNIEWICZ B., SACHSE-KOZLOWSKA E., KUBIAK H. 1975 - Górnopaleolityczne stanowisko Kraków-ul.Spadzista C (badania w latach 1970-1973). Folia Quatemaria, 45.

LANCZOT M., Boguckyj A., 2002 - Badane profile lessowe i stanowiska paleolityczne. Studia Geologica Polonica, 119, 33-181.

LINDNER L., 1992 - Czwartorzed. Osady, metody bada, stratygrafia. Warszawa:Wydawnictwo PAE.
MADEYSKA T.(ed), 2002 - Lessy i paleolit Naddniestrza Halickiego. Studia Geologica Polonica, 119.

MANIA D. 1981 - Eine Fundstelle des PerigordienGravettien von Bilzingsleben. Archaeologia Interregionalis, vol.1, 89-94.

NOIRET P. 2003 - Le Paléolithique supérieur de la Moldavie. Essai de synthèse d'une évolution multiculture Ile. Thèse de doctorat, Université de Liège.

OTTE M., NOIRET P. 2004 - Evolution du Gravettien du Danube moyen. In : The Gravettian along the Danube, B rno:Institute of Archaeology AS CR, 8-32.

OSTROVSKIY M.I., GRIGORIEV G.P. 1966 Lipskaya paleoliticheskaya kultura. Sovietskaya Arkheologia, 4, 9-16.

PAZDUR A., GOSLAR T., MICHCZYNSKI A., PAWLYTA J. 1999 - Zastosowanie metody radioweglowej do datowania ósadow mlodszego czwratorzedu. In : Geochronologia Górnego Czwartorzedu Polski, Gliwice:Politechnika Slaska, 16-42.

PEAN S., WOJTAL P. 2003 - Gravettian subsitance patterns related to Pleniglacial palaeoenvironments in Southem Poland. In : Le rôle de l'environnement dans les comportements des chasseurs-cueilleurs préhistoriques. BAR International Series, 1105, 23-37.

PIASETSKIY V.K. 1991 - Paleoliticheskoye mestonakhojdenie Jornov; verkhniy kulturnyj sloy. Rossiyiskaya Arkheologia, 2.

PIASETSKIY V.K. 2001 - Verkhnepaleoliticheskaya stoyanka Gradki v vostochnoy chasti volynskoy vozvychennosti. Arkheologitcheski Almanach, 10,45-54.

PLONKA T. et WISNIEWSKI A. 2004 - New Gravettian site in Lower Silesia. In : The Gravettian along the Danube. Brno:Institute of Archaeology AS CR, 164-179.

SAVICH V.P.1975 - Paleolitichne naselennia pivdennozakhidnoy Volyni. Kiev:Naoukova Doumka.

SITNIK O. 1998 - Do pitannia pro "lipsku piznopaleolitichnu kulturu" na Volyni. In : Minule i Souchastne Volyni. Lutsk, 192-196.

SITNIK O., BOGUTSKIY A. et MADEYSKA T. 1996 Startifikovani pamiatki paleolitu $v$ okolitsach Galicha. Arkheologia, 3, 86-97.

SOFFER O. 1993 - Upper Palaeolithic adaptations in Central and Eastern Europe and man-mammoth interactions. In : From Kostenki to Clovis, New York:Planum Press, 31-49.

SRODON A., MAMAKOWA K. 1977 - O pleniglacjalnej florze z Nowej Huty i osadach czwarto rzedowych doliny Wisly 
pod Krakowem. Rocznik Polskiego Towarzystwa Geologicznego, 47, 4, 485-511.

SVOBODA J. 2002 - Afterwords: the Pavlovian as a part of Gravettian mosaic. In : The Gravettian along the Danube, B mo:Institute of Archaeology AS CR, 283-297.

TERBERGER T., STREET M. 2002 - Hiatus or continuity?New results for the question of Pleniglacial settlement in Central Europe. Antiquity, 76, 691-698.

VALDE-NOWAK P. 2000 - The boomerang from Oblazowa and its prehistoric content. In : La chasse dans la prehistoire, ERAUL, 51, 88-94.

VALDE-NOWAK P. 2003 - Oblazowa Cave: new light on Gargas hands. International Newsletteron Rock Art. , 35, 7-10.

VALDE-NOWAK P., NADACHOWSKI A., MADEYSKA T. (eds) 2003 - Oblazowa Cave, human activity, stratigraphy and palaeoenvironment.Krakow:Institute of Archaeology and Ethnology PAS.
VAN VLIET B. 1988 - Le rôle de la glace de ségrégation dans les formations superficielles de l'Europe de l'ouest. Processus et héritages. Université Paris I, thèse de doctorat.

VAN VLIET B. 1992 - Le niveau a langues de Kesselt. Horizon repère de la stratigraphie du Weichselien supérieur européen: signification paléo-environnementale et paléoclimatique. Mémoire de la Société Géologique de France, $160,35-44$

WEST D. 1996 - Krakow-Spadzista, unit E and F. Faunal Remains. Folia Quaternaria, 67, 21-34.

WOJTAL P., CYREK K., SITNIK A. 2001 - The new Upper Palaeolithic mammoth site at Halitch (Ukraine). Acta Zoologica Cracoviensia, 44, 2, 137-142.

WOJTAL P., SOBCZYK K. 2005 - Man and wolly mammoth at the Krakow Spadzista Street (B) - taphonomy of the site. Joumal of Archaeological Science, 32, 193-206. 
